\title{
Forces During Cell Adhesion and Spreading: Implications for Cellular Homeostasis
}

\author{
Shawn P. Carey, Jonathan M. Charest and Cynthia A. Reinhart-King
}

\begin{abstract}
Cells adhere and spread by exerting forces against the cell membrane and against the extracellular matrix. Intracellular forces drive the membrane outward during spreading and stabilize cell shape in adherent and migrating cells. A balance of intracellular force with exogenous forces is required for maintenance of basic cell functions and cellular homeostasis. Here, we provide a multi-scale overview of the cellular machinery and intracellular forces at play during cell spreading and adhesion, including description at the molecular, cellular and tissue levels. We describe the cellular machinery required for force generation, explain aspects of its regulation, and show how the machinery operates to direct a cell to a homeostatic target. The biochemical and biophysical events that dominate the process of isotropic cell spreading are examined and the process of spreading is explained as a series of distinct phases, each with their own force signature. In addition, we consider how intracellular force affects mechanical cellular homeostasis and maintenance of tissue structure and function. The disruption of cellular mechanical homeostasis is described in the context of two prominent disease states: cancer and atherosclerosis.
\end{abstract}

\section{Introduction}

Current research has brought much attention and interest to the role of cell mechanics in cellular homeostasis and physiological function. Cell adhesion and

\footnotetext{
S. P. Carey, J. M. Charest and C. A. Reinhart-King ( $\square)$

Department of Biomedical Engineering, Cornell University, 302 Weill Hall, Ithaca, NY 14853, USA

e-mail: cak57@cornell.edu
} 
spreading is a primary mechanism through which mechanical (and sometimes chemical) homeostasis is achieved. These processes are crucial in both development and the maintenance of normal tissue structure and function. As such, improper cell adhesion and spreading has been implicated in a multitude of disease states, including cancer and atherosclerosis. Here we will take an in-depth look at the processes of cell adhesion and spreading, with a specific emphasis on the role of cell mediated-force: first describing the subcellular machinery employed during adhesion and spreading, then describing the cellular level changes of a single cell through the process, and finally examining how disruption of cell adhesion and spreading can lead to disease.

\section{Biological Machinery Required for Force Generation}

\subsection{Internal Cellular Machinery}

Cellular forces are primarily generated in the cytoskeleton. The cytoskeleton is essential for maintaining cell shape and organization, imparting specific mechanical properties to cells. There are three primary cytoskeletal components: microfilaments, microtubules, and intermediate filaments. While each component serves separate functions in the cell, there are several commonalities:

- Each component is composed of repeating subunits that polymerize to form polarized filamentous structures.

- Polymerization of most components requires the binding of either ATP or GTP to its subunits.

- Each polymerized component has characteristic mechanical properties that are directly determined by the structure of its subunits.

- Polymerized structures associate with various other structural and signaling proteins for purposes such as stabilization, regulation, or linkage to other parts of the cell.

- Each component has associated motor proteins that facilitate relative axial movement in a stepwise fashion dependent upon ATPase activity, contributing to the generation of cellular forces.

Here we focus primarily on microfilaments and microtubules, as they are the primary cytoskeletal filaments associated with cellular force generation.

\subsubsection{Actin and Myosin}

Actin is the primary component of the cytoskeleton required for the generation of cellular forces imposed externally and is by far the most studied in the context of the regulation of cell spreading and adhesion. In a cell, actin can generate forces 
through two primary mechanisms: (1) simply through polymerization or (2) through coupling to its associated motor protein, myosin.

\section{Actin Structure and Dynamics}

Actin can exist in two distinct forms within the cell: globular actin (G-actin) or filamentous actin (F-actin). G-actin is a $\sim 43 \mathrm{kDa}$ protein that can bind ATP and polymerize to form microfilaments known as F-actin. The nucleation of F-actin filaments is controlled and accelerated by certain chaperone proteins such as Arp $2 / 3$ among others. An F-actin filament is polarized and has a positive $(+)$ end and a negative $(-)$ end. Both ends are highly dynamic but have different on/off rates; growth of filaments occurs primarily at the positive end, as it has a much higher on rate. Actin filaments are highly cross-linked and bundled by proteins such as $\alpha$ actinin to increase their structural integrity. Actin dynamics and the two mechanisms through which it can generate force are summarized in Fig. 1. Figure 1a, b depicts the polymerization of actin which can drive membrane extension. Figure 1c depicts actin's interaction with myosin which can drive filament sliding and the generation of contractile force.

\section{Actin-Interacting Proteins}

The cellular actin network owes its highly dynamic nature to the actin-interacting proteins that facilitate its assembly, stabilization, and disassembly. The protein

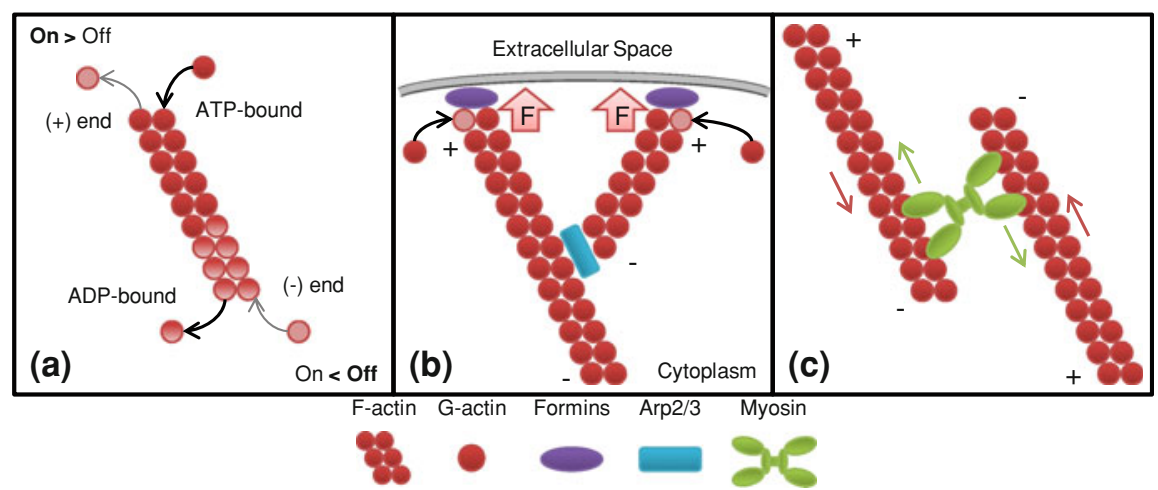

Fig. 1 Actin filament-based force generation. a Filament dynamics: filament polymerization and depolymerization occur at opposite ends resulting in filament "treadmilling". b Force generation through cortical branching at the cell membrane: Arp2/3 mediates microfilament branching and formins mediate actin polymerization at the cell membrane producing protrusive forces. c Myosin-mediated contraction of microfilaments: myosin mediates the contraction of anti-parallel overlapping microfilaments, the mechanism at the core of cell contractile force generation. Outer arrows indicate direction of filament displacement, inner arrows indicate direction of myosin motor displacement 
complex Arp2/3 facilitates actin filament nucleation, enables branching by attaching to a filament and nucleating a new filament and aids in network stabilization. Filament polymerization can be enhanced by the family of proteins known as formins and the protein VASP [1]. Formins act as "processive caps" by attaching the $(+)$ end of the filament to the plasma membrane while simultaneously enabling further polymerization and protecting the filaments from other capping proteins [2]. Complementary to these proteins are ADF/cofilin, which disassembles actin from its (-) end [1]. Another group of important actin-interacting proteins are those that link actin to transmembrane adhesion molecules (discussed in later sections of this chapter). The linkages between actin and the membrane involve a large, multi-component complex of proteins that facilitate transmission of chemical and mechanical signals between the intracellular and extracellular space. Talin is largely implicated as one of the critical structural proteins linking f-actin filaments to transmembrane integrins [3, 4]. These transmembrane complexes are particularly important as they allow actin-generated forces to be transmitted to the cell's environment.

\section{Measurement of Actin-Generated Forces and Mechanical Properties}

During cell adhesion and spreading, forces generated by actin polymerization can be exerted directly against the cell membrane. McGrath and colleagues showed that actin polymerization is capable of exerting forces on either curved or flat surfaces, based on their data which shows that the force of actin polymerization can propel micron-sized synthetic disks through solution [5]. These findings implicated that actin polymerization can exert force on a substrate regardless of its curvature. This is particularly important in the context of cell generated forces, as actin polymerization forces are primarily exerted on the highly fluid cell membrane.

Only relatively recently has the force of actin polymerization been quantitatively measured $[1,6]$. The force generated by a branching actin network was first investigated by Fletcher and colleagues in 2005 [6] using atomic force microscopy (AFM). An AFM cantilever tip was coated with ActA (an actin-interacting protein from Listeria monocytogenes that facilitates polymerization), placed into cell extract, and an actin network was allowed to form (Fig. 2a). The AFM cantilever tip was then used to measure the displacement of, and force exerted on, the growing actin network. The data is depicted using a force-velocity $\left(F_{v}\right)$ curve as shown in Fig. 2d.

$F_{v}$ curves are used to depict the force profile in biological systems because experimentally, force in biological systems is often measured using optical methods to track displacement against an opposing force. These opposing forces can be provided by objects such as a micron-sized bead in a viscous suspension [5], or an AFM cantilever tip as was the case in these experiments [6, 18]. These measurements of actin polymerization from Fletcher and colleagues [6] revealed two distinct phases of force generation: a load-independent phase and a stalling 


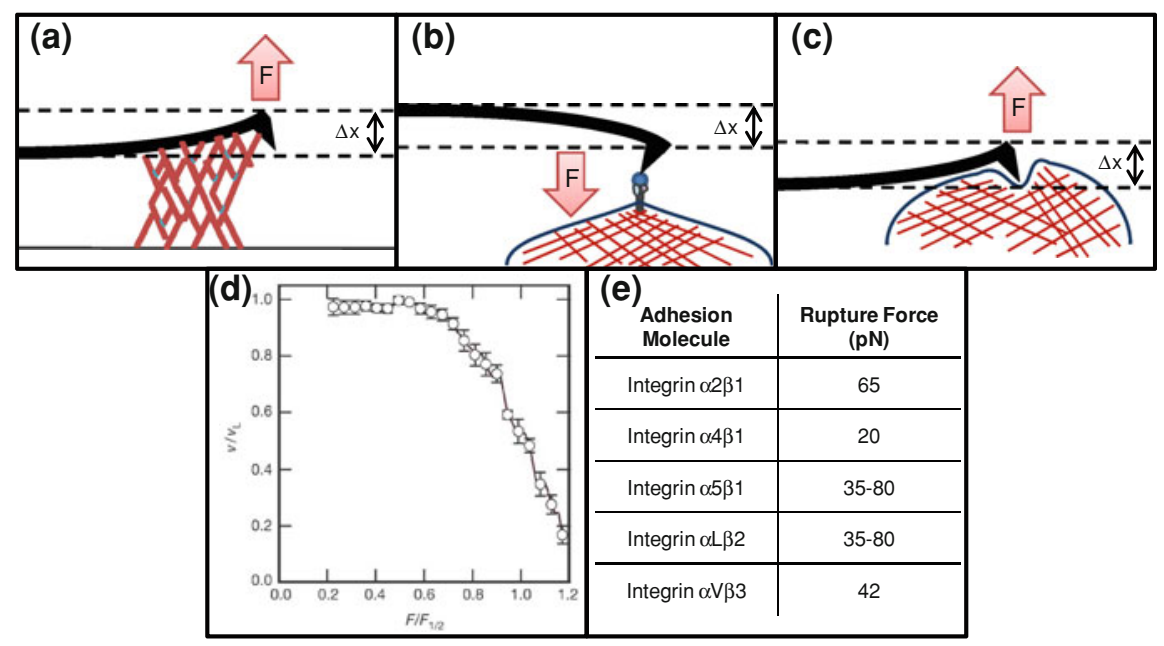

Fig. 2 Methods of measuring cellular level forces. a Actin polymerization force against an AFM cantilever tip causes tip displacement. b Integrin ligands are attached to an AFM cantilever tip, then brought into contact with the cell surface. The tip is then displaced away from the cell until the ligated integrins are torn from the membrane. $\mathbf{c}$ Lamellipod protrusion force against an AFM cantilever tip causes tip displacement. d Reprinted by permission from Macmillan Publishers Ltd: Nature Cell Biology [6], copyright 2005. Normalized $F_{v}$ curve of actin polymerization obtained through method depicted in a [6]. e Single-cell rupture forces for various integrins using the experimental setup depicted in $\mathbf{c}$. Ranges are given when more than one value has been reported [7-17]

phase. The load-independent phase indicated that the network was capable of growing at a relatively constant rate over a fairly large range of forces, about 0-300 $\mathrm{nN}$. The stall force (the force at which velocity reaches zero) measured for the network of actin filaments is on the order of hundreds of nano-newtons. Additional studies have used AFM to measure the force exerted by actin polymerization in a protruding lamellipod [18] (Fig. 2b). Data from these experiments illustrates that actin polymerization is the driving mechanism of protrusive force generation in cells, and is critical to cell spreading.

Microfilament rigidity is also critical to the ability of actin fibers to provide stable cellular structure and effectively transmit force. One measure of the stiffness of a given cytoskeletal filament is its persistence length, which represents the rough distance over which a filament may be considered completely rigid. Persistence lengths of actin filaments have been measured by observing their deformation under thermal fluctuations or various loading conditions [19-22]. Early studies concluded that the persistence length of an actin filament is around $17 \mu \mathrm{m}$ [19]. Later studies examined filaments under different conditions (nucleotide binding, phalloidin binding, etc.) and found the persistence length could vary from 7 to $20 \mu \mathrm{m}$ [22]. Additionally, actin-interacting proteins such as cofilin have also been shown to influence filament persistence length [21]. The cell's ability to modulate microfilament persistence length is important in fine-tuning force generation. 


\section{Myosin Structure and Dynamics}

The myosins are a family of motor proteins associated with actin. Actin-myosin complexes generate the cellular forces used in cell contractility and migration. The majority of myosins are $(+)$ end motors, meaning that they move along actin filaments towards the (+) end. There are several different types of myosins and each participates in specific cellular functions. In this chapter, we will be primarily concerned with non-muscle myosin II (NMII), as it is most often implicated in the generation of cellular-level forces [23]. Myosin "heavy chains" consist of one or more head, neck, and tail domains. Head domains bind actin and require ATP to detach. As such, if ATP is depleted within a cell, its myosin molecules remain attached to actin and cannot move. The neck domain contains binding sites for myosin "light chains" (calmodulins) that regulate the head domain. Activation of myosins such as NMII requires phosphorylation of its light chain domain. The tail domain facilitates the interaction of myosin proteins with each other, forming myosins with more than one head and allowing the formation of myosin filaments (a common example of this type of filament can be found in the A-band in the sarcomere of skeletal muscle). Functionally, myosins also strengthen the actin network by cross-linking actin fibers. Studies of myosin molecule interactions with actin fibers using optical trapping techniques indicated that a single myosin molecule is capable of exerting forces on the order of $\sim 3.5 \mathrm{pN}$ while producing a displacement of about $11 \mathrm{~nm}$ [24].

\subsubsection{Microtubules}

In comparison to actin, microtubules are much larger, more rigid [19], and slightly less dynamic. Although their role in cell adhesion and spreading is less understood, it is known that they contribute to the mechanical properties of cells and are involved in the establishment of cellular homeostasis. Like actin, microtubules have specific associated motors that contribute to force generation. However, we will not specifically discuss the microtubule-associated motor proteins (kinesins and dyneins) as they function primarily for transport within the cell and are not directly involved in the generation of cellular-level, adhesion-related forces.

\section{Microtubule Structure and Dynamics}

Microtubule subunits are heterodimers made up of the globular proteins $\alpha$-tubulin and $\beta$-tubulin. Their structure is complex: $\alpha / \beta$-tubulin dimers first polymerize longitudinally to form protofilaments. The protofilaments then line up side by side to form curved sheets that eventually wrap into a tube consisting of 13 protofilaments in circumference. Both $\alpha$ - and $\beta$-tubulin require GTP in order to form a dimer and polymerize into full microtubules. Nucleation and stabilization of long microtubules is mediated by $\gamma$-tubulin and its associated proteins (known as the $\gamma$-tubulin ring 
complex, or $\gamma$-TuRC). In the cell, microtubules generally originate from the microtubule organizing center (MTOC), which is found near the centrioles.

Like actin, microtubules are polarized and have both a positive end (which is highly dynamic) and a negative end. Assembly and disassembly occurs primarily at the microtubule (+) end. The assembly rate is lower than the disassembly rate, primarily because disassembly can occur in a "catastrophic" fashion. Conformational changes of the subunits occur when the $\beta$-tubulin in the $\alpha / \beta$-tubulin heterodimer hydrolyzes its bound GTP to GDP, creating a "kink" between the $\alpha$ - and $\beta$-tubulin. This kink can cause unstable microtubule ends to disassemble by peeling apart.

\section{Measurement of Microtubule-Generated Forces and Mechanical Properties}

Similar to actin dynamics, both microtubule polymerization and depolymerization are known to generate force. Early studies of microtubule polymerization forces analyzed the buckling of microtubules against an immobile barrier to calculate an $F_{v}$ curve [25]. These studies found an exponential decay relationship in the $F_{v}$ curve, closely resembling modeling predictions. Subsequent attempts to observe microtubule assembly dynamics used more advanced techniques such as optical traps, enabling molecular-level resolution [26]. Methods to measure microtubule persistence length are similar to those used to examine actin filaments. As mentioned earlier in this chapter, microtubules have a much higher rigidity than actin filaments. This is evidenced in the relatively high persistence length of microtubules, which is on the order of about $5 \mathrm{~mm}$ (as compared to actin filaments, at around 10-20 $\mu \mathrm{m}$ ) [19]. This also fits well with observations that microtubules generally support compressive loads within the cell [27, 28].

\subsubsection{Intermediate Filaments}

Intermediate filaments are the third major class of cytoskeletal elements. As a part of the cytoskeletal network, they contribute to cellular shape and structural integrity, and are largely considered cellular "shock-absorbers" to mediate and mitigate external mechanical stresses [29]. The class of cytoskeleton proteins known as intermediate filaments is really a collection of various proteins, each with different tissue specificities, but a conserved filamentous structure. Depending on the specific filament type, intermediate filaments can have persistence lengths ranging from $200 \mathrm{~nm}$ (keratin) to $1,300 \mathrm{~nm}$ (vimentin) [30]. Therefore intermediate filament proteins are far softer and more flexible than microtubules and actin filaments. While it is not known if intermediate filaments plan an active role in adhesion and spreading, it is clear they play a mechanical role in the cell by acting as a stress-buffering system. A more extensive review of the structure and mechanical properties of intermediate filaments can be found in the following extensive reviews [29, 30]. 


\subsection{Cell-Environment Connection Machinery}

The ability of cells to generate forces through the intracellular machinery described above is critical for a variety of essential cellular functions. Intimately tied to the generation of forces is the ability of a cell to attach to its matrix in order to transmit and stabilize these intracellular forces.

Early studies showed that cell-matrix adhesions are crucial for cell survival $[31,32]$ and established the concept of "anchorage dependence," referring to the need for a cell to attach to a substrate to survive. Landmark studies using the then newly-purified ECM protein fibronectin revealed the presence of a physical transmembrane link between the specific constituents of the ECM and the cytoskeleton. For example, treatment of transformed cells with fibronectin caused "increased cell-to-substratum adhesion of treated cells, cell spreading and flattening, and elongation of cell processes" [33] as well as reorganization of cytoskeletal F-actin [34]. It was observed that extracellular matrix macromolecules such as fibronectin aggregate into filaments and serve as ligands for specific transmembrane receptors, which cluster and serve as docking stations for intracellular cytoskeleton proteins such as actin [35-37]. Shortly thereafter, the phenomenon of cellular "shape sensing" was identified as the basis for density dependent cell growth inhibition and anchorage dependence [38]. This concept has since grown significantly and implications of this work are important for our understanding of cell adhesion, spreading, and homeostasis.

\subsubsection{Integrins}

The transmembrane receptors that mediate the dynamic physical interactions between cytoskeleton-extracellular matrix were first purified in 1986 and were termed integrins $[39,40]$. Integrins are heterodimeric ( $\alpha$ - and $\beta$-subunits), transmembrane proteins with highly conserved extracellular and cytosolic domains. These transmembrane macromolecules are being studied in a wide number of human health-related fields, including development, cancer, hematology, and immunology. In the past three decades, the integrin family has expanded to include a number of homologous adhesion receptors whose most basic function is to bind to insoluble extracellular matrix protein ligands. However, their role is more complex, as they mediate both physical and chemical interactions through their involvement in focal adhesions. Additionally, their signaling functions are bidirectional, capable of both "inside-out" and "outside-in" signaling.

\section{Integrin Specificity}

Integrins bind to specific extracellular matrix glycoproteins to allow a cell to adhere, spread, and migrate. The combination of integrin subunits imparts specificity. 
Certain cells and their integrins adhere only to specific matrix proteins. Inherent in this is ligand-specific "outside-in" signaling. While integrins bind specific sequences in matrix proteins, integrins do not demonstrate singularity. That is, a given integrin (an $\alpha / \beta$ combination) can potentially bind with several different extracellular matrix ligands. For example, the $\alpha 5 \beta 1$ integrin can act as the adhesion receptor for both fibronectin and collagen. Therefore, adhesion and spreading of a given cell-type are mediated through cell-type specific integrins ligating their corresponding matrix proteins.

Integrin Avidity, Affinity, and Activation

Also important in cell adhesion and spreading is a cell's ability to control and modulate integrin function. This ultimately determines how tightly a cell can adhere to a substrate in its microenvironment. Studies with leukocytes and platelets provided evidence that some cells can change their adhesiveness in response to microenvironmental factors [41]. Changes in cellular adhesiveness or "avidity" do not occur through recruitment of receptors to the membrane, but rather through increased integrin affinity (through integrin activation) and valency (governed by density of receptor and ligand on surfaces). Regulation of integrin affinity through intermolecular interactions and intramolecular allostery is an elegant demonstration of the bi-directionality of integrin signaling.

- "Inside-out" signaling:

- Short cytoplasmic integrin tails (primarily of the $\beta$-subunit) interact with membrane-associated intracellular proteins.

- The ligand-binding site is restructured through long-range allosteric conformational changes that result in increased integrin affinity (or "activation").

- "Outside-in" signaling:

- Subsequent ligand binding causes conformational changes that are transmitted back to the cell to regulate diverse responses [42].

This "activation" process can also run in reverse, beginning with integrins binding to extracellular ligands and resulting in the presentation of cytoskeletal adapter protein binding sites. Integrin avidity can also be impacted by valency: increased integrin clustering, increased membrane diffusivity of $\beta$ subunits [43]. As is often the case with integrins, movement of integrin receptors laterally within a membrane can be initiated both extracellularly (as a result of extracellular ligand availability) and/or intracellularly (through F-actin-mediated relocation of integrins) [44]. Integrin organization within the plasma membrane has several implications in terms of both signaling and internal cytoskeletal organization, which will become more apparent in later sections. 
Mechanical Characterization of Integrin Interactions

Even though integrins themselves are incapable of generating force, they are essential components in transmitting cell-generated forces to the environment. To achieve this, integrins must be capable of withstanding relatively high levels of mechanical stress. Studies to determine the amount of force integrins can withstand before failing are vast [45], although they generally fall into two categories: whole cell studies and single integrin studies. AFM has been a useful tool in characterizing integrin binding affinities. Using AFM in this setting allows a substrate to be brought into contact with the cell surface and then removed with known displacement and force (Fig. 2c). Using this technique on the single cell level has revealed a rupture force (force at which the ligand unbinds from the receptor) on the order of $30-80 \mathrm{pN}$, given a loading rate of $1 \mathrm{nN} \mathrm{s}^{-1}$ [45]. A more comprehensive review of using AFM to measure cellular binding interactions appears in a recent paper by Helenius et al. [45].

Optical traps (or "optical tweezers") have also been used extensively to measure adhesion molecule load bearing [45]. This method involves using micronsized beads coated with integrin ligands. These beads can be controlled using optical techniques and used to exert force on a cell once in contact with its adhesion receptors. Galbraith and colleagues used this method to investigate how forces exerted on integrins mediate focal complex formation, a concept we will discuss in more detail in Sect. 4 [44, 46].

\subsection{Cell-Cell Connection Machinery}

Just as cells have transmembrane integrins to connect their internal machinery to their environment, they also have transmembrane machinery specifically for establishing connections with other cells. These molecules follow the same theme of exhibiting a high degree of specificity in their function, enabling cells to sense their environment. Cell-cell contact also regulates the extent of spreading and cell force generation [47].

The primary mediators of cell-cell adhesion are the cadherin family of proteins. Cadherins are glycoproteins with a single transmembrane domain. There are several types of cadherins, E-, N-, and P-cadherin, and their distribution is tissuespecific. Their extracellular domains (N-terminal) bind other cadherins in a homotypic manner (i.e. a E-cadherin molecule will bind only other E-cadherins) that is $\mathrm{Ca}^{2+}$-dependent. The cytoplasmic domains (C-terminal) link to the actin and microtubules of the cytoskeleton with the help of $\alpha$ - and $\beta$-catenin. Dimerization of cadherin molecules is also required for cell-cell adhesion. E-cadherin is the prototypic cadherin and has been implicated in several signaling pathways relating to the establishment of cell polarity, actin polymerization, and Rho activation.

Another family of cell-cell connection molecules is the immunoglobulin (Ig) superfamily. This family includes molecules such as N-CAM, ICAM, VCAM, 
PECAM and several others. Like the cadherins, they are transmembrane glycoproteins, however they bind in a heterotypic manner and are not dependent on $\mathrm{Ca}^{2+}$ for their ligation. Expression of these molecules is tissue-specific and can be induced by certain cytokines.

Yet another group of transmembrane glycoproteins involved in cell-cell connections are the selectins. This family has several members (E-Selectin, P-Selectin, L-Selectin). Expression is cell type specific and in some cases can be induced by exposure to growth factors such as TNF $\alpha$. These heterotypically bind sugar moieties on the surface of other cells in a $\mathrm{Ca}^{2+}$-dependent manner.

The cell-cell adhesion molecules, once engaged, are most commonly found clustered in the membrane, similar to the way integrins cluster. Cell-cell junctions mediated by cadherins linked to actin and myosin filaments are collectively known as adherens junctions (AJs). Like matrix adhesions, these sites include proteins such as vinculin, tropomyosin, and $\alpha$-actinin (many of the same components as focal adhesions). A second type of cell-cell junction is the desmosome. This type of cell junction consists of a cytoplasmic plaque (made up of plakoglobin and desmoplakins) that links keratin fibers (intermediate filaments) to desmoglein and desmocollin, which are both cadherins. These adhesions are thought to contribute to a cell's shape and rigidity. They may also be able to transmit shear forces from one cell to its neighbors.

Cell-cell connection machinery provides yet another pathway through which cells can sense their environment to reach their homeostatic target. In the context of cell spreading, this target takes the form of contact inhibition, a phenomena in which cells will stop spreading if they come into contact with neighboring cells. Such interactions are crucial to normal tissue function, but are disrupted in certain disease states as will be described in Sect. 5 .

\section{Subcellular Connections and Dynamics}

\subsection{Matrix Adhesions}

As described in the previous section, a cell possesses machinery for internal force generation through cytoskeletal polymerization and machinery for exerting force against its extracellular matrix. Coupling of these two separate but related mechanisms to generate force enables cell-matrix adhesion and cell spreading. Here we discuss the regulation of this coupling and how it translates into a means for cells to sense their environment.

\subsubsection{Initiation and Formation}

Cell-matrix adhesion formation begins with the ligation of an adhesion receptor. This initiates an "outside-in" signal-usually through conformational change of 
the adhesion receptor-that alerts the cell to its new connection. Adhesion-associated scaffolding and signaling proteins are then recruited to the new adhesion site. These recruited proteins often serve multiple purposes ranging from physical reinforcement to propagation of chemical signals and regulation of the adhesion lifetime. Important to their function, the activities of these proteins are transient; the regulatory events governing matrix adhesions and cytoskeletal reorganization require the molecules involved to be intrinsically dynamic. The development and maturation of matrix adhesions is often dependent upon the exertion of forces at the adhesion site.

Focal adhesions are commonly found in cells spread out on a 2D substrate and their formation is based around the ligation of integrins to their specific substrate in the ECM. Focal adhesions are molecular assemblies containing clusters of multiple scaffolding and signaling proteins [48, 49] which are typically elongated adhesions that are greater than $1 \mu \mathrm{m}^{2}$ in area. Focal adhesions mature from focal contacts, which are small dot-like adhesions, typically after the application of force [50]. One of the first proteins to be recruited by a ligated integrin is talin. Talin binds directly to the $\beta$-subunits of integrins and serves as a platform for both linking to the cytoskeleton via actin filaments and binding to other recruited proteins such as paxillin (also an integrin-binding protein) and vinculin [51]. Together, talin and paxillin recruit focal adhesion kinase (FAK). FAK has multiple functions within the cell, including contributing to focal adhesion turnover, transduction of signals to regulatory proteins, and phosphorylation of other focal adhesion proteins [3]. Src, a membrane-associated tyrosine kinase, forms a complex with FAK that aids in further signal propagation [3]. The transient formation of this complex and its subsequent intracellular signaling play a role in determining the lifetime of the focal adhesion. Contractile force imposed at adhesion sites and changes in cell shape cause the clustering of additional ligated integrins and their respective linkages to the cytoskeleton [44]. This force contributes to the maintenance and stability of adhesion sites required for cell adhesion and spreading.

\subsection{Mechanosensing at the Cell-Matrix Interface}

The inside $\rightarrow$ outside $\rightarrow$ inside mechanosensory ability of cells is a critical controller of not only cell adhesion and spreading, but also of endogenous cellular force generation, tissue homeostasis, and quiescence [52]. Moreover, the ability of a cell to respond to external mechanical stimuli depends on highly-interconnected and coordinated networks of regulatory signaling that are integrated in the same pathways that regulate cell adhesion. In their role as signaling and structural transmembrane complexes, integrin-mediated complexes have mechanosensing 
function and are capable of translating externally applied forces into cellular phenotypic changes.

Although no single sensory molecule has been identified specifically for the purpose of mechanotransduction (as has been identified for chemical stimuli, for example), mechanical stimuli in the cellular microenvironment including exogenous forces, physical properties of the ECM, and forces exerted through cell-cell adhesions have a significant effect on cell behavior. It has been shown that cells can sense external forces through a number of mechanisms, including primary cilia, stretch-modulated ion channels, nuclear lamina, the cytoskeleton, and matrix adhesions [53-55]. Cellular adhesion, spreading, and force generation rely primarily on cytoskeletal dynamics and matrix adhesions as a mechanism to sense and respond to externally applied mechanical forces.

The ability of cells to sense the stiffness of their environment, while an emerging field, is becoming a classic biological example of cellular mechanotransduction resulting in differential cell spreading. Stiffness sensing relies upon "active tactile exploration" of the cellular microenvironment. That is, cells use cytoskeletal contractility to exert contractile forces through matrix adhesions, assessing the resulting substrate strains to determine the appropriate cellular response. While this phenomenon was originally observed and identified in the context of cell morphology, motility, and adhesion strengthening [56, 57], ongoing work has shown that mechanosensing of matrix stiffness is intimately involved in directional cell migration [58], cell differentiation [59], tissue development and maintenance [60-65], disease progression [66, 67], and tensional homeostasis [66, 68].

The maturation and development of matrix adhesions into focal adhesions during cell spreading and adhesion is directly affected by substrate stiffness. Initial cell adhesions to the ECM through integrins initiate integrated feedback and feedforward signaling cascades that actively regulate cell adhesion and cell force generation [69]. Mechanosensing is a driving force for this response, and it has been shown that stable focal adhesions, as evidenced molecularly by an adhesionlocalized increase in tyrosine phosphorylation and behaviorally by decreased membrane ruffling and rate of spreading, only develop on sufficiently stiff substrates [56]. Cells perceive stiffness by interpreting the reactive tensile force that results from contracting the ECM through adhesions; less stiff (more compliant) substrates resist less tension, and thus, do not promote the cytoskeletal tension and protein kinase phosphorylation that are required for focal adhesion maturation and stability $[44,48]$.

Cells use adaptive matrix adhesions to integrate mechanical cues from their microenvironment. Through recruitment of adapter proteins and signaling molecules to sites of initial matrix adhesions, cells can perceive and respond to exogenous forces as well as physical properties of the extracellular matrix. In the following sections we will demonstrate how cells utilize integrated biochemical and biophysical pathways to adhere, spread, change shape, and migrate by providing analysis of the phases of cell spreading. 


\section{A Spreading Cell}

Thus far, we have laid the groundwork for understanding the dynamics of cell adhesion and spreading by describing the cellular structures (cytoskeleton and integrin-mediated adhesions) involved. Given this information, the specific process of cell spreading can be dissected. Cell adhesion and cell spreading are fundamental biological processes essential for establishing connections between a cell's internal machinery and its environment. The establishment of these connections, or lack thereof, will dictate cell behavior by enabling a cell to sense and respond to its environment. These connections are therefore critical for achieving cellular homeostasis.

Cells have been shown to spread either isotropically, where they flatten against a surface like pancake batter on a pan, or anisotropically, where the cells send out membrane extensions unevenly in multiple directions [64]. In earlier work, we showed that when ligand density is decreased, anisotropic spreading is favored; however, when ligand density on a substrate is saturated, isotropic spreading is favored [64]. While there remains much to be understood regarding the mechanisms driving isotropic versus anistropic spreading, our data seem to indicate that the need for a cell to "search" for ligand to bind to, when ligand is sparsely coated on a surface, triggers a cell to send out increased extensions, which favors anisotropic spreading. The rate and directionality of filopodia extension depending on the microenvironment may be one determinant of the mode of spreading.

Although most cell types display both modes of spreading, current literature has primarily focused on isotropic spreading [70-76]. Spreading studies, in general, are useful model systems for simplifying the more complex process of cytoskeletal organization, and isotropic spreading is the simplest form of that model. Spreading can be thought of as a series of cytoskeletal movements that drive membrane protrusion. Given this simplification, spreading becomes a relatively pure system for understanding the forces and factors regulating cytoskeletal reorganization. Once these aspects of spreading are understood, this knowledge can be applied to our understanding of a more complicated cell processes like migration or cell division, where multiple cytoskeletal processes, like adhesion, de-adhesion, ruffling, blebbing, and retraction, occur.

Here, we will dissect the process of cell adhesion and spreading, by describing the changes undergone by a single cell from initial contact to a fully spread state and the establishment of homeostasis. To do this however, we must first make some assumptions:

- The cell is healthy, anchorage-dependent, and spreads isotropically (equally in all directions).

- The substrate exhibits sufficient adhesion molecule ligands on its surface. These ligands can take the form of ECM proteins such as fibronectin, laminin, collagen, etc. 
Thus, the specific phases of spreading described here may not apply to all cell types, but because all eukaryotic organisms, including yeast, utilize similar cytoskeletal proteins and dynamics, one might anticipate that cell spreading generally occurs in unique phases. Isotropic spreading is focused on here because it much simpler mechanistically. Anisotropic spreading involves the same mechanisms, except they are spatially biased.

\subsection{Defining the Phases of Spreading}

Much of what is known about the individual phases of cell spreading is largely based on the work of only a few labs in recent year. Sheetz and colleagues were the first group to define the distinct phases involved in cell spreading [72, 73]. These phases were identified by comparing the spread area $(A)$ of isotropicallyspreading mouse embryonic fibroblasts on fibronectin-coated substrates with time $(t)$. Examining a plot of $\ln (A)$ vs. $\ln (t)$ revealed three distinct rates of area increase, each following a different power law (and thus a different "rate" of spreading, $d A / d t$ ). Generally, cells exhibit an initially slow rate of spreading, followed by a period of rapid spreading, before finally reaching a fully spread state with minimal change in area. Upon examining cellular traction forces over the same duration, a biphasic trend emerges: traction forces remain low for a period of time before abruptly rising to higher levels later on, once a constant area is achieved. An example of this behavior can be seen in Fig. 3. The time course over which spreading occurs can vary depending on cell type. However, the phases of spreading as identified [72, 73] occur over the course of roughly an hour, as seen in Fig. 3.
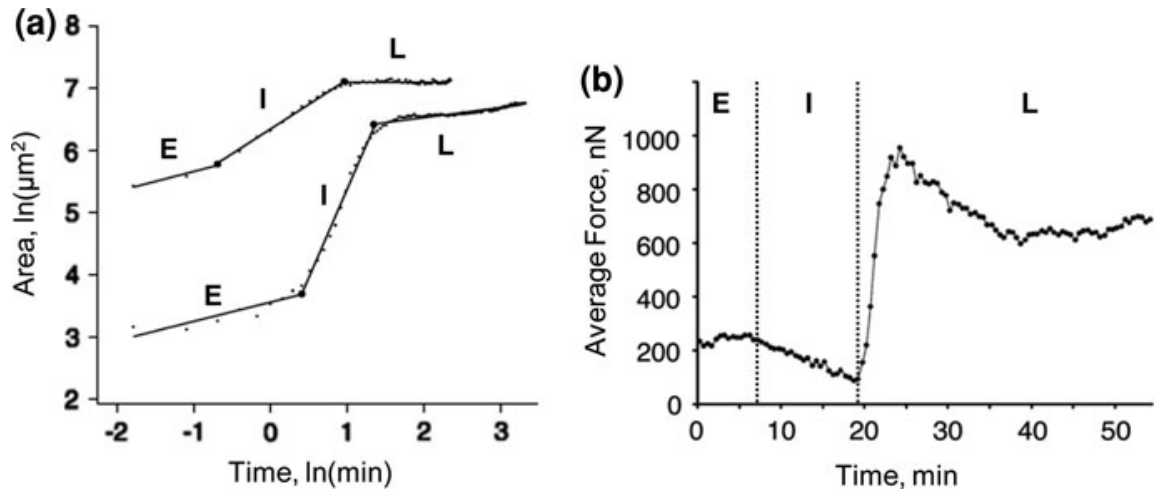

Fig. 3 Identifying the phases of spreading (a, reprinted with permission from the Journal of Applied Physiology) double logarithmic plot of cell area over time for two different mouse embryonic fibroblasts [73]. b Average contractile force of a single spreading mouse embryonic fibroblast over time [76] (Reproduced under Creative Commons Attribution License). Dotted lines indicate boundaries between phases. $E, I$, and $L$ indicate early, intermediate, and late phases of spreading respectively 
Integrating these observations with knowledge of cytoskeletal structure and function provides some information regarding the intracellular dynamics involved in spreading. As described earlier in this chapter, a major function of the cytoskeleton is to establish and support cell shape, structure and integrity. Multiple groups of proteins (such as small GTPases) actively regulate cytoskeletal dynamics (actin/microtubule (de)polymerization, myosin activation/inhibition, etc.). These regulatory proteins directly control cell shape, structure, and integrity by regulating the cytoskeleton. A cell's degree of spreading-and more broadly, its morphology - at any given time is thus the collective combination of all active cytoskeletal "states" or activity $[72,73]$. Precise spatial and temporal activation of cytoskeletal states gives rise to cellular processes (membrane blebbing, ruffling, lamellipodia or filopodia extension, contraction, etc.) that are then coordinated or biased to achieve global movements such as adhesion, spreading, migration, etc. [76]. Investigation of the molecular players involved in each phase of cell spreading should provide valuable insight into the relative roles that each of these molecules play in regulating more complex processes like migration.

\subsection{Phases of Spreading}

\subsubsection{Early Phase Spreading}

\section{Key Events}

The early phase of spreading can be thought of as containing two key events: first is the initial physical contact between the cell and substrate, followed by the initial sensing of the substrate surface ligands. In most cases, cells initially exhibit a spherical or "balled-up" morphology while in suspension.

\section{Initial Physical Contact with Substrate}

The dynamics of the initial physical contact between the cell and substrate is governed primarily by the material properties of both entities [74, 75]. Very early spreading and the initial forces experienced by a cell can be analogously compared to a viscous liquid droplet (the cell) contacting a surface (the substrate). This very early spreading is primarily a passive process, dependent upon the mesh-like structure of the cortical actin network of the cell and the stiffness of the cell membrane [74, 75]. The cortical actin network can increase cell rigidity and decrease initial passive spreading. As such, disruption of the cortical actin network (through disrupting either actin polymerization [74], or non-muscle myosin-IIA (NMIIA) contractility [77]) significantly increases the rate at which early spreading occurs by decreasing the amount of force required to deform the cytoskeleton-supported cell membrane. Another material aspect important in 
early contact with the substrate is surface charge. The surface of a cell is normally negatively charged due to carbohydrate modifications of surface proteins. As such, the early adhesion dynamics between cell and substrate depend on the thermodynamic balance of charges on each. Predictive models of these cellsubstrate interactions in terms of such thermodynamics have been developed $[78,79]$.

\section{Initial Sensing of Substrate Surface}

Coincident with the initial physical contact of the cell and its substrate are the formation of the initial connections between cellular adhesion receptors (predominantly integrins) and substrate-bound extracellular matrix proteins. Membrane-bound adhesion receptors are brought into further contact with the substrate via active cytoskeletal protrusion events in early spreading. These events include either membrane blebbing or filopodia extension [76]. These first connections are significant events because they serve as both anchoring points for the cell facilitating adhesion, and as nexuses to transmit information regarding the composition of the cell substrate. This ability arises from the specificity of its adhesion receptors (e.g. specific integrin subunit pairs bind only specific ligands).

\section{Implications of Early Phase Events}

The establishment of initial cell-substrate adhesions characterizes the early phase of spreading, and will ultimately induce a transition to the next phase of spreading. Activation of integrins, via binding to their extracellular ligands, initiates multiple signaling events that can influence cytoskeletal dynamics and the maturation of the initial adhesions.

Transduction of integrin-mediated signals typically requires the intracellular binding of paxillin and talin, which recruit focal adhesion kinase (FAK) and Src. FAK and Src form a complex together to initiate a cascade of signaling that results in stimulation of the Rho GTPases Rac1 and Cdc42. Activity of this FAK-Src complex also mediates suppression of active RhoA (RhoA bound to GTP) levels, which in turn decreases the activity of Rho-associated kinase (ROCK), thereby decreasing cell contractility $[3,80]$. Paxillin and talin also serve to link the ligated integrin to the cytoskeleton. Together, increased activity of Rac1 and Cdc42 serve to stabilize the F-actin network of the cell and promote its growth through increased polymerization [80], which is the driving mechanism of the next phase of spreading described below [72, 73, 76].

The density of ligand available on the substrate has been shown to affect the duration of this early phase of spreading [44, 76, 81]; cells initiate spreading more quickly on a surface containing higher ligand density. Likely, a cell exposed to an increased density of initial ligand will exhibit increased early 
integrin activation that results in activation of Rac1 and Cdc42. At the end of early phase spreading, the cell has transformed from being a spheroid in suspension, to being a flattened cylinder with roughly the same radius on the $2 \mathrm{D}$ substrate.

\section{Cell-Generated Forces During Early Phase Spreading}

Studies have shown that a cell at this early stage is capable of exerting contractile forces on the substrate [76, 81]. Contractile forces at this stage, however, are of significantly lower magnitude as compared to later stages of spreading. The generation of early phase contractile forces, shown to be caused by NMIIA [77], is enabled by the linkage of ligated integrins to the cytoskeleton via talin [4]. These weaker contractile forces are to be expected, as RhoA activity is suppressed by the formation of initial adhesions and that the cell's small adherent spread area can only minimally activate ROCK to induce myosin contractility [82]. Our own work has shown that cells are capable of exerting force in these early stages of spreading, even without the detectable presence of actin stress fibers or the clustering of vinculin, one of the earliest recruited focal adhesion-associated proteins [64]. Collectively, these data indicate that to exert force against its substrate, a cell does not need formal focal adhesions to have formed.

Summary: Early Phase spreading is characterized by:

- Passive deformation and disruption of the cortical cytoskeleton occurs, dependent on the stiffness and tension of the cell membrane.

- The formation of initial adhesions between integrins and extracellular matrix proteins, which initiates signaling events through Rac1, Cdc42, and RhoA. Integrin ligation alters the balance of active cytoskeletal processes.

- Weak contractile forces exerted against the substrate, likely due to minimal integrin binding and the lack of focal contacts.

\subsubsection{Intermediate Phase}

\section{Key Events in Intermediate Phase}

The cell enters the intermediate phase of spreading once Rac1 and Cdc42 have been activated beyond a threshold, essentially reached through ligation of adhesion receptors to the substrate $[72,73,76,80]$. This increased activation of Rac1 and Cdc42 shift the dominant active cytoskeletal state from a primarily stagnant cortical network to polymerization of the cortical actin. Therefore cell area rapidly increases during the intermediate phase of spreading. 


\section{Implications of Intermediate Phase Events}

Increased peripheral actin polymerization results in an increase in protrusive forces, causing a rapid increase in cell area. Rac1- and Cdc42-induced actin polymerization drives an increase in cell area by exerting protrusive forces directly on the membrane [1]. During intermediate phase spreading, VASP localizes near the tips of cortical protrusive processes [76]. This helps drive expansion of cell area because VASP binds both F-actin and adhesion molecules in the cell membrane [83, 84]. A number of additional actin-interacting proteins including the Arp $2 / 3$ complex, formins, and cofilin also play a role in actin polymerization and the protrusion of the cell membrane during spreading. Formins act as "processive caps" by attaching the (+) end of the filament to the plasma membrane while simultaneously enabling further polymerization and protecting the filaments from other capping proteins. The Arp2/3 complex enables branching of the actin network, providing increased stabilization between filaments. To support the expanding cortical actin network, depolymerization of older actin filaments must occur. Depolymerization is mediated by the cofilins, which aid in the dissociation of ADP from G-actin. In the next phase of spreading, actin-interacting proteins (formins, Arp2/3, VASP, and talin) also play a large role in shaping the ability of microfilaments to exert and transmit force. The retrograde flow of actin has been shown to be inversely related to the speed of edge protrusion and driven by a combination of NMIIA activity and membrane resistance to actin polymerization $[70,85,86]$.

The increase in cell area, which is a hallmark of this intermediate phase of spreading, also causes several other notable changes to occur in the cell. One of the more obvious changes is the increase in the number of integrin-mediated adhesions. As spread area increases, more of the cell surface comes in contact with the substrate, and more integrin receptors become ligated. Integrin ligation initiates the formation of a positive feedback loop via Rac1 and Cdc42 [80]. Just as occurs during early phase spreading, initial integrin ligation activates these regulatory small GTPases. The intermediate phase of spreading also includes an increase in cytoskeletal tension. Studies of cells on isolated adhesive islands of ECM revealed that cells with a greater spread area exhibit higher levels of cytoskeletal tension [87]. Increased cytoskeletal tension translates to increased intracellular force transmitted to the cell-matrix adhesions, which balances the internally-generated forces across the cell. Increased tension at adhesion sites activates additional signaling pathways inducing activation of RhoA, a key molecular regulator of contractility [82].

Increased protrusion forces also induce the recycling of adhesion sites. As spreading occurs and new adhesion sites are formed, mature adhesion sites (now located towards the center of the cell body) begin to disassemble due to prolonged FAK activation and decreased RhoA activity [70, 88]. Recall that the initial ligation of integrins causes deactivation of RhoA and a number of other events through FAK activation [3]. Integrins from disassembled adhesion sites are now recycled to new sites at the cell periphery [86]. The recycling of integrins has been 
linked to ROCK signaling [89] which can become decoupled from RhoA activity [82] (such that RhoA activation does not activate ROCK). Through this pathway, adhesion site disassembly leads to the majority of adhesion sites being located at the expanding cell periphery.

\section{Cell-Generated Forces During Intermediate Phase Spreading}

As described earlier, cell-generated forces in intermediate phase spreading are predominantly protrusive. These protrusive forces are driven by actin polymerization directly against the membrane and are facilitated by specific actininteracting proteins [1]. Protrusive forces have been measured using a variety of tools. The first direct measurement of these forces was in 2006 by Prass et al. [18]. They measured the deflection of an AFM cantilever tip against the advancement of a trout keratocyte lamellipod and used that measurement to calculate the force exerted by the lamellipod. Using this technique, the mean force produced by lamellipodial protrusive forces was determined to be just over $1 \mathrm{nN}$ [18]. The normalized $F_{v}$ curves obtained in their experiments exhibited the same qualities as the $F_{v}$ curve for simple actin polymerization as measured by Parekh et al. [6] in Figure $2 \mathrm{~d}$, illustrating that such lamellipodial protrusive forces are generated through actin polymerization.

As in early phase spreading, there appears to be weak contractile force generation during this phase due to minimally active ROCK [82]. Together, the increase in cell area, recycling of adhesion molecules, and increased tension induce onset of the third, "late" phase of spreading. The shift is driven primarily by the increased activation of RhoA and its downstream effector ROCK, induced by the cell reaching a critical spread area [71, 82].

Summary: Intermediate Phase cell spreading is characterized by:

- "Steady state" phase of actin polymerization [76], resulting in the generation of protrusive forces at the cell periphery and generation of new adhesion sites.

- Increased cytoskeletal tension.

- Recycling of adhesion receptors from initial locations to the cell periphery.

\subsubsection{Late Phase}

\section{Key Events in Late Phase Spreading}

Similar to the Intermediate Phase of spreading, the onset of Late Phase spreading is induced by an increase in the levels of activated small GTPases involved in contractility. Once the cell's spread area reaches a critical spread area (as determined by substrate properties such as ligand density, stiffness, etc.) [71, 81], RhoA activates its effector ROCK, resulting in NMIIA phosphorylation. This increase in 
phosphorylated NMIIA produces the hallmark event of late phase spreading: cytoskeletal contractility.

\section{Implications of Late Phase Events}

The resultant increase in RhoA activation and its coupling to ROCK [82] leads to the activation of myosin light chain kinase (MLCK) which in turn activates NMIIA via phosphorylation. Increased NMIIA activation mediates contraction of the cytoskeleton and transmission of the subsequent generated force to the substrate. As in intermediate spreading, the key characteristic event, cell contraction, promotes several changes in the cell. Most notably, contractile forces imposed at sites of cell-matrix adhesion induce reorganization of adhesion molecules and reinforcement of adhesion sites.

First, the increased force on ligated integrins promotes integrin clustering in the membrane and the maturation of focal adhesions [90]. Clustering of integrins and activated RhoA leads to bundling and cross-linking of the associated actin and myosin network into stress fibers. RhoA also serves to inhibit actin fiber disassembly by cofilins and promote its assembly via formins (the "processive caps" described earlier in this chapter) [91].

Contractile "pulses" of the cytoskeleton are also observed in Late Phase spreading. These contractile pulses have been proposed as a way for cells to "sense" substrate stiffness [70, 76].

\section{Cell-Generated Forces in Late Spreading}

Late phase spreading is characterized by a rapid increase in cell-generated contractile forces [71, 76]. The degree of cell contractility (and the magnitude of generated forces) is affected by substrate stiffness, ligand concentration, cell area, focal adhesion size, and a variety of other factors [71, 76, 81]. The contractility observed in late phase spreading is transmitted through integrin adhesion to the cell substrate as tractional stresses exerted tangential to the substrate. Multiple techniques have been developed to measure cellular traction stresses, including traction force microscopy (TFM). TFM measures the cell-mediated displacement of fluorescent beads in a deformable substrate of known stiffness to determine forces exerted by a cell that creates these displacements. Studies using TFM have revealed that endothelial cells can exert traction forces almost as high as 2,500 $\mathrm{nN}$ $[81,92]$. Cells can therefore exert much greater force through contractile mechanisms in late spreading compared to polymerization-driven mechanisms in early spreading.

Summary: Late Phase spreading is characterized by:

- NMIIA-dependent contractility.

- Formation of actin-myosin based stress fibers linked to mature focal adhesions (Fig. 4). 


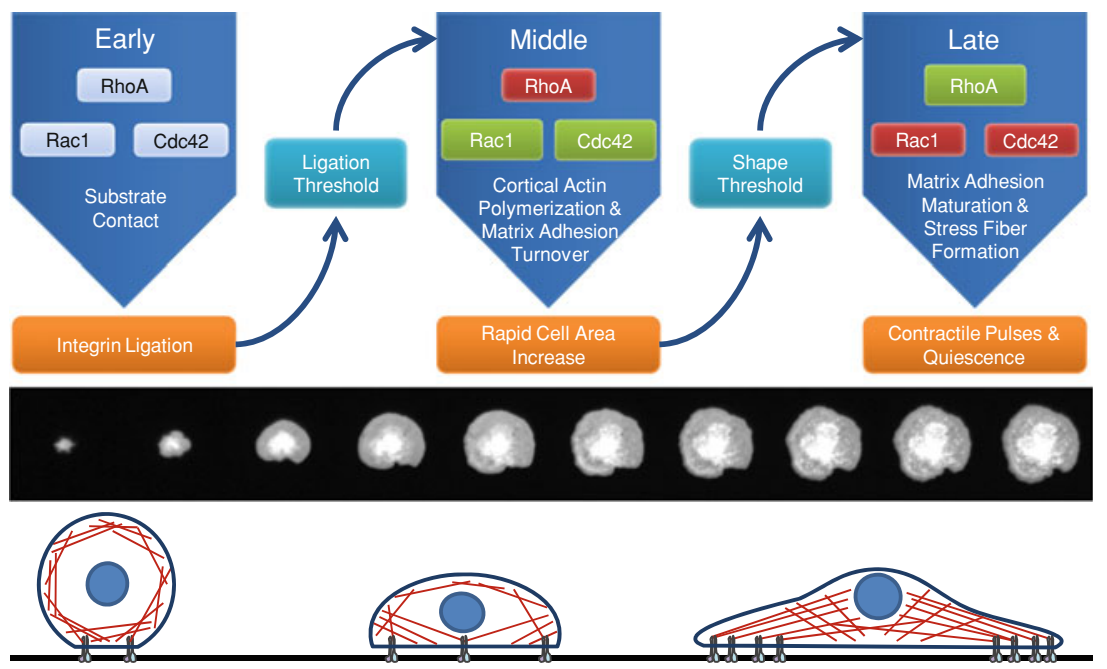

Fig. 4 A summary of the phases of isotropic cell spreading. Top panel Flowchart of spreading events; activated and inactivated Rho GTPases are indicated by green and red boxes, respectively; orange boxes indicate the events that directly lead to initiation of the next phase of spreading by crossing the threshold indicated in the teal boxes. Middle panel Total internal reflection fluorescence (TIRF) microscopy images of the spread area of a mouse embryonic fibroblast over time [76] (Reproduced under Creative Commons Attribution License). Bottom panel Cartoon rendition of cell spreading events, viewed from the side

\section{Physiologic Environmental Factors Sculpt Cellular Spreading and Homeostasis}

\subsection{Regulation of Cell Shape by the Extracellular Microenvironment}

During active and quiescent cell states, the multi-molecular intracellular machinery that is required for force responsiveness and generation is not static. Rather, the cytoskeleton and cell-matrix adhesion complexes are continually being assembled and disassembled, enabling the cell to generate protrusive and contractile forces. These forces contribute to the maintenance of cellular homeostasis in a potentially dynamic microenvironment, and during cell migration and the formation of tissues [49, 93-95]. In the previous section, this coordination was demonstrated through the phenomenon of isotropic cell spreading, which is generally considered a relatively homogeneous process. Physiologically, however, there are many environmental factors that influence cell spreading and determine the homeostatic shape of a cell. For example, the cellular microenvironment can affect cell contractility, kinetics of spreading, spread area, cell polarization (intracellular components), and isotropy of shape and cell elongation. Notably, while these parameters generally define cell morphology, cell shape is much more 
significant than a mere description of the size and appearance of a cell. Over the past 30 years, several landmark studies in which the geometry of cells was precisely controlled have proven that cell shape, as determined by cell-matrix adhesions and the cytoskeleton, has a critical role in cell survival and differentiation as well as in tissue development, architecture, and function in vivo [38, 96-99]. Identifying the environmental factors that contribute to defining cell shape in vivo will enable better understanding of cell and tissue homeostasis as well as the causes and effects of deregulated cell shape in disease.

\subsection{Cell Migration: "Shape Change with a Bias"}

Cell migration is a dynamic process that occurs through the biochemical and biophysical orchestration of cell-matrix adhesions and cell shape polarization, which itself is a coordination between intracellular contractile and protrusive forces [100-103]. Thus, in the context of cell adhesion and spreading, cell migration can be considered "shape change with a bias". Indeed, the extracellular stimuli that drive cell shape regulation often direct cell migration in tissue development, maintenance, and disease [95, 104, 105]. Such microenvironmental factors can influence migratory characteristics including rate of migration (speed) and directional persistence in a coordinated manner. Highly-polarized stimuli, such as chemical or mechanical gradients, will typically increase the directional persistence of a migrating cell while generally isotropic stimuli, such as uniform chemokinetic factors or ECM stiffness, will alter the migratory behavior (speed) of a cell with no apparent directional bias. While a complete discussion of the mechanisms of cell migration is beyond the scope of this chapter, we will discuss how integration of exogenous stimuli drives endogenous cellular force generation, thus enabling cells to adaptively control their shape and, as necessary, migrate to achieve cellular homeostasis.

\subsection{Tissue Specificity}

Mesenchymal, connective, muscle, epithelial, and endothelial cells are each exposed to unique microenvironmental factors in vivo that define distinctive cell and tissue morphologies and functions [106-111]. It would be impossible to provide a comprehensive survey of instances where a cell's microenvironment defines its cellular homeostasis within one chapter, as research in this field is vast and entirely tissue-specific. However, to capture the importance of the microenvironment in regulating cellular shape and force generation, two specific tissue types will be discussed: epithelial and endothelial tissues. The environmental factors that contribute to the unique homeostasis of the cells in each tissue will be explored. We will provide evidence that, through mechanosensing, the microenvironment 
mediates endothelial and epithelial cellular force generation, which, when orchestrated with matrix adhesions, manifest in cell shape regulation. Finally, this shape regulation, when spatiotemporally coordinated, can lead to cell migration, which has implications in tissue development and disease progression.

\subsection{Overview of Cellular Homeostatic Perturbations and Disease}

Cell shape changes and migration, which are required for embryogenesis, tissue maintenance, and wound healing, are also involved in pathogenesis upon disruption of cellular homeostasis. Perturbations in cell spreading can cause cells to undergo changes in gene expression and phenotype that drive it from its quiescent state. Normal disruption of homeostasis is transient and can be beneficial as it guides cell differentiation and migration during development or wound healing. However, sustained disruption of homeostasis or aberrant cell response to homeostatic microenvironmental conditions can drive disease. If the homeostatic perturbation is sustained and/or excessive, it can initiate and preserve overactive intracellular signaling loops that cause tissue-specific phenotypic changes and promote pathogenesis. For example, changes in ECM stiffness, which are typically a sustained perturbation, can cause deregulation of cell-generated forces, which, as we will discuss, can significantly impact the normal structure and function of cells and tissues. Additionally, if the ability of a cell to respond to environmental cues is abnormal, its behavior in its native environment may be disrupted. For example, transformed cells can have defective mechanosensing machinery, which prevents them from sensing and responding to their mechanical environment appropriately.

\subsubsection{Epithelium and Cancer: An Example of Cell Forces Correlating with Disease}

Epithelial tissues exist in several unique shapes, including spherical cysts (i.e. thyroid, other glands) and elongated tubular structures (i.e. ducts in mammary epithelial tissue) [112]. Regardless, the most striking characteristics of epithelial tissues in vivo are their apical-basal polarity and tight cell-cell adhesions [113, 114]. Notably, the tissue stroma has been strongly implicated in establishing these traits. Further, the properties and composition of both the cellular and ECM components of the stroma evolve through development and significantly affect tissue organization and function at all epithelial generations [108, 115]. It has been shown that cytoskeletal tension, controlled through Rho and ROCK-mediated contractility, drives basement membrane remodeling required for normal tubulogenesis in mammary epithelium [116, 117]. Additionally, several studies have demonstrated that proper epithelial development and maintenance depends on a 
dynamic balance between cell-cell and cell-matrix adhesions [95, 118]. Guo et al. [63] found that cell-cell adhesions compete directly with cell-matrix adhesions to provide cells with mechanical input and thus, maintain tensional homeostasis. If physical signals from the ECM are stronger than those from cell-cell interactions, cells migrate away from each other, while if cell-cell adhesions dominate, cells associate and assemble into tissue structures. This balance of epithelial cell adhesions - to the basal lamina through integrins or to other cells through E-cadherins - plays a critical role in establishing epithelial tissue polarity. The nature of cell adhesions promotes polarization of the membrane and cytoskeletal molecules across the apical and basal cell surfaces [113].

\section{Basal Lamina Stiffness Drives the Malignant Phenotype Through a Cellular Force Pathway}

The stroma of epithelial tissues stiffens naturally during both tissue morphogenesis and aging [119]. Naturally occurring tissue stiffening is due to the accumulation of advanced glycation end products which crosslink the matrix as well as increased matrix deposition. It is also well-known that tumors are stiffer than the surrounding stromal tissue. This stiffening is likely a result of increased interstitial tissue pressure and solid stress due to tumor expansion [120], an increase in the intracellular stiffness of malignant cells [121], excessive RhoA-based cell contractility [122, 123], and fibrosis [105]. Furthermore, patients presenting clinically with fibrotic tumors typically have a poor prognosis [124]. Because of the highly interconnected feedforward/feedback nature of mechanosensing, matrix stiffness can initiate and maintain chronically-elevated mechanoresponsive signaling cascades that can lead to malignant transformation of epithelial cells.

Stiffness sensing is an important component of a cell's mechanosensing arsenal, and it has been identified as a major determiner of epithelial cell shape and epithelial tissue homeostasis. Epithelial cells probe the stiffness of the surrounding ECM and respond through the adhesion and cytoskeletal dynamics discussed throughout this chapter [112]. As discussed in earlier sections, matrix stiffness induces focal adhesion formation and stability through an increase in tension at these adhesion sites. This phenomenon is especially important in epithelial tissues, where the balance between cell-cell and cell-matrix adhesions is controlled primarily by properties of the underlying basal lamina [112, 115]. Thus, epithelial tissue architecture is governed in part by the stiffness of ECM and cellular force generation, and increases in stiffness can induce dissociation of cell-cell adhesions [63]. Such deregulation of cell-cell adhesions supports an increased preference for cell-matrix adhesions through integrins, which is critical for cancer progression. In fact, inhibition of $\beta 1$ integrins in breast cancer cells arrested cell growth and promoted cellular apoptosis in 3D cultures and in vivo [125], underscoring the necessity of cell-matrix adhesions and cell spreading in cell survival.

During malignancy and invasion, cells exhibit a more aggressive phenotype, and it is thought that cell force generation is an important element of this 
phenotype [126]. Notably, epithelial cell traction forces increase with matrix stiffness [127], which can manifest in basement membrane reorganization, cell shape changes, and migration commonly associated with cancer $[115,117,128]$. Indeed, the expression and activity of the small GTPases Rac and Rho, which are classically linked with cellular contractility, are enhanced in tumors, and elevated Rho expression is clinically associated with cancer progression $[122,129,130]$. Further, Wozniak et al. [116] have implicated Rho-based cell contractility in response to matrix stiffness as the determining factor that drives mammary epithelial cells toward either tubulogenesis on soft substrates or cell proliferation on stiff substrates. Rac and Rho have complementary roles in maintaining cell shape and cell force generation. Cancer cells, which typically exhibit loss of spatiotemporal control over Rac and Rho activation, show disrupted cell polarity, cell shape, and cell force generation [131, 132].

Taken together, the deregulation of cell adhesions, increase in cell force generation, and cell shape changes that occur in response to basement membrane stiffness provide substantial evidence that normal levels of ECM stiffness preserve epithelial tissue architecture (Fig. 5). Several groups have shown that a sustained increase in stromal stiffness disrupts normal cellular and tissue structure and function both in vitro and in vivo, in a manner that is characteristic of cancer. Paszek et al. [66] demonstrated that increased stiffness causes integrin clustering, focal adhesion assembly, disruption of E-cadherin junctions, Rho-mediated contractility, and FAK/ERK activation in non-transformed mammary epithelial cells. These subcellular effects resulted in disruption of acini structure and promoted a highly invasive phenotype. Moreover, Provenzano et al. [67] showed that sustained ERK activation due to matrix stiffness promoted initiation of, and progression through, the cell cycle as well as expression of clinically-relevant proliferationsignature genes, which is consistent with the characteristic over-proliferative behavior of malignant cells. Most recently, it was reported that stiffening of the mammary stroma with lysyl oxidase (a natural collagen crosslinker) facilitates tissue morphology breakdown and invasiveness both in vitro and in vivo [133].

The matrix stiffness-induced intracellular changes shown by Paszek et al. and Provenzano et al. may enable epithelial to mesenchymal transition (EMT), which is generally characterized by fundamental changes in cell adhesion and motility $[95,134]$. Specifically, cells having undergone EMT exhibit a loss of E-cadherin expression in favor of $\mathrm{N}$-cadherin expression. These cell-cell adhesions are much weaker, and an increase in cell-matrix adhesions is needed to satisfy the cells' need for mechanical input and tensional homeostasis.

In addition to triggering malignant transformation, stiffness-induced Rhomediated cell contractility and changes in cell adhesion, when coupled with proteolysis, may also contribute to metastatic migration [135]. This "shape change with a bias" is likely enabled by defective mechanosensing components (discussed in the next section), and allows tumor cells to navigate aggressively away from the primary tumor. While a more thorough discussion of the mechanisms of cell migration is outside the focus of this chapter, Friedl and Wolf [103] provide an excellent review. 


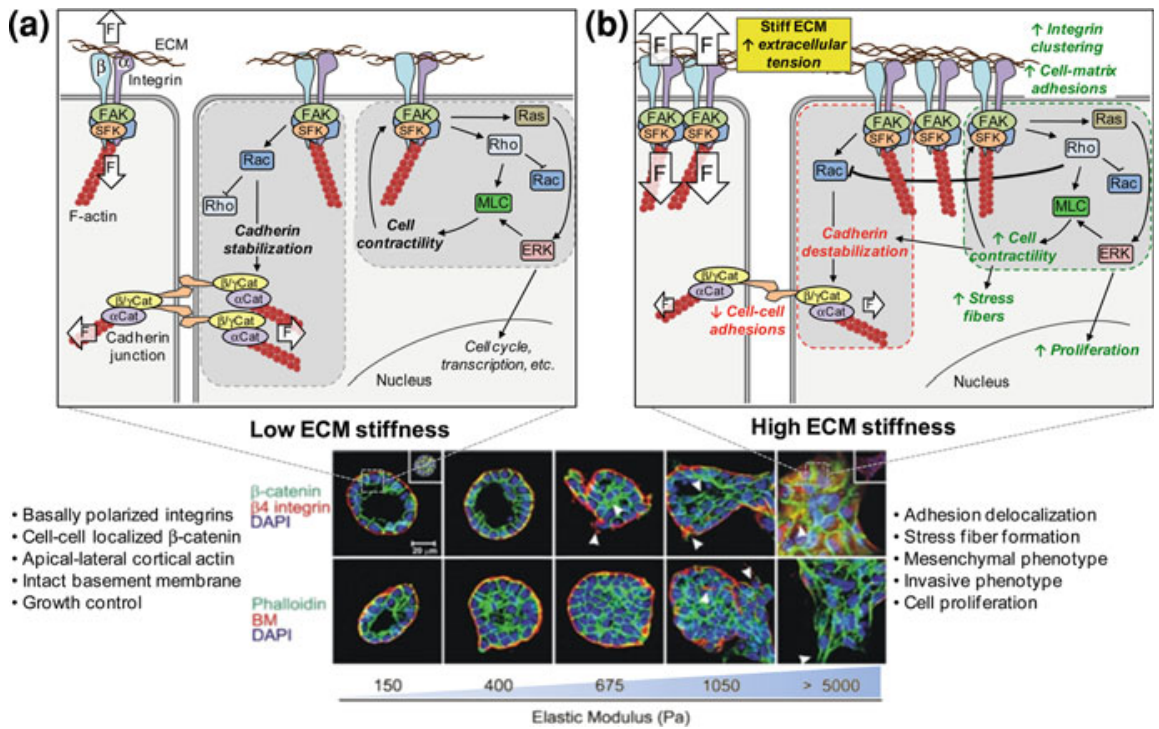

Fig. 5 Normal ECM stiffness promotes epithelial cell quiescence and epithelial tissue structure. a Spatiotemporally-localized activation of Ras, Rac, and Rho downstream of FAK phosphorylation contributes to epithelial cell polarity and maintenance of tensional homeostasis under normal ECM conditions (left side of figure). Specifically, Rac acts to stabilize cell-cell junctions through recruitment of cortical actin (dashed gray region on left), while activated Rho and ERK phosphorylate MLC and drive cell contractility (dashed gray region on right). Both cell-generated and perceived extracellular forces are represented by white arrows. b In response to a stiff extracellular matrix (right side of figure), the cell re-establishes its tensional homeostasis by contracting more aggressively (positive feedback pathway within green dashed region), which enables stress fiber formation and promotes cell-matrix adhesion through integrin clustering. The impact of stiffnessinduced Rho activation on intercellular cadherin junctions is twofold (destructive signaling pathway within red dashed region). Rho directly suppresses (thick black inhibition arrow) the laterallylocalized Rac activation that stabilized cadherin junctions and, through cell contractility, destabilizes already existing intercellular junctions. ECM stiffness promotes a general loss of cellular polarity, which drives the epithelial cells toward an invasive mesenchymal phenotype. Together, the subcellular and cellular-level responses in green and red are indicative of a malignant phenotype and the resulting disruption of normal tissue architecture is represented in the confocal immunofluorescence images, with notable events highlighted by white arrowheads. Confocal immunofluorescence images reprinted with permission from Elsevier [66]

\section{Defective Mechanosensing Components Prevent Epithelial Cells from Responding Appropriately to Mechanical Stimuli}

The ability of cells to respond to their environment is critical for maintenance of cellular health and homeostasis. However, the intracellular signaling pathways that are involved in sensing and responding to external stimuli are complex and involve a vast number of molecular players. Gain-of-function mutations in Ras that result in its constitutive activation were among the first discovered oncogenic mutations and represent one of the most prevalent molecular mechanisms for transformation [136]. Overactivation of this molecular intermediate has many downstream effects, 
one of which is decreased sensitivity to the mechanical properties of the surrounding ECM. Transformed cells typically exhibit increased cell contractility [66, 134], which is driven by altered RhoGTPase signaling in breast cancer [123]. This defective mechanosensing capability was modeled in vitro by transfecting mammary epithelial cells with a constitutively active isoform of Rho. This modification eliminated the stiffness-sensing ability of these cells, causing elevated cell contractility, increased cell spreading, and enhanced cell-matrix adhesion regardless of matrix stiffness [66].

\section{Mechanical Properties of the ECM Influence Angiogenesis in Cancer}

Normal development and maintenance of endothelial tissues also depends on mechanosensing, balance of cell-cell and cell-matrix adhesions, and coordination of contractile and protrusive cytoskeletal dynamics. Interestingly, there is significant evidence that blood vessels associated with tumors, and specifically those that develop within tumors through angiogenesis, are leaky, dilated, and disorganized, and that the cells that reside in these blood vessels are highly proliferative, drug-resistant, and exhibit increased sensitivity to growth factors $[137,138]$.

The process of neovessel formation through angiogenesis is an essential part of development, wound healing, and pathogenesis [139]. There is evidence that proteolytic activity accompanies angiogenic network formation in vivo, suggesting that matrix composition affects both the initiation and progression of neovessel formation [140]. Furthermore, because sprouting involves directional migration, it requires polarization, which is thought to arise from both biochemical and biophysical means. The ECM plays a significant role in establishing the cell polarization that accompanies endothelial network formation. Through force generation and mechanosensing, cells detect and respond to intrinsic properties of the ECM as well as changes in these properties.

During angiogenic network formation, cell-cell adhesions can directly compete with cell-matrix adhesions [63] to enable the self-assembly of cells into networks structures. This balance supports the formation of networks in more compliant ECM, where to satisfy their tensional homeostasis, endothelial cells increase cellcell interactions and connectivity [62, 64]. Our own laboratory has showed that when EC-matrix adhesivity is low, either through insufficient matrix stiffness or reduced ligand availability, capillary-like network formation results (Fig. 6) [47]. Alternatively, when EC-matrix adhesions are strong and stable on stiffer substrates, the cells are less likely to organize into networks. While this phenomenon was identified in vitro, it has significant implications in tissue development and maintenance in vivo, particularly in driving endothelial sprouting and network formation during tumor angiogenesis. Specifically, these results suggest that increased intratumoral stiffness may cause the destabilization of endothelial cellcell adhesions, resulting in excessive vascular permeability [138].

Additionally, we have acquired separate data to show that ECs are capable of mechanosensing adjacent cells over a distance during network formation. That is, 


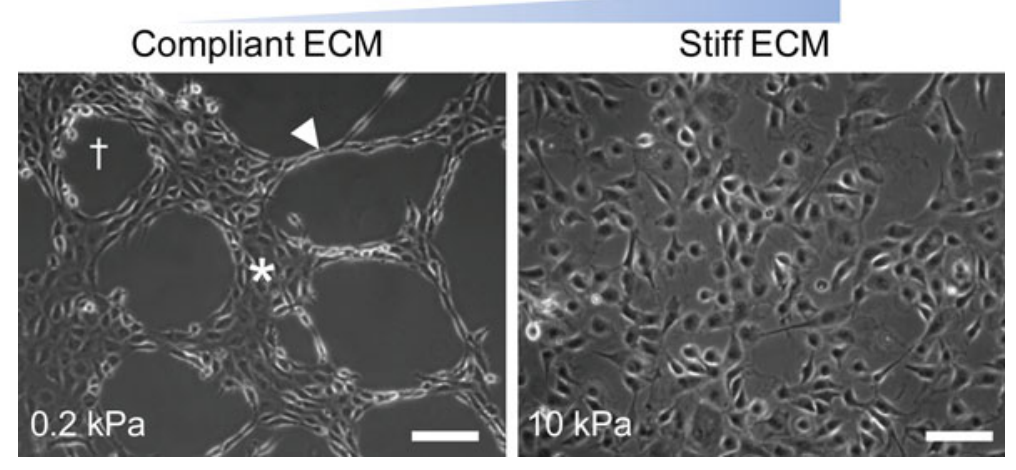

Fig. 6 Matrix stiffness influences endothelial network formation. Compliant (low stiffness) gels ( $E \leq 1 \mathrm{kPa}$ ) promote cell-cell interactions and network assembly as evidenced by EC clusters, cords, and lacunae. Stiff gels $(E \geq 2.5 \mathrm{kPa})$ prevent proper endothelial network formation by restricting endothelial cells from deforming their ECM to communicate with other cells as well as by encouraging cell-matrix adhesions. This phenomenon is important in cancer because the ability of tumor-associated ECs to satisfy their tensional homeostasis through balance of mechanical inputs is critical for normal vascular function. Increased intratumoral stiffness may cause destabilization of cell-cell adhesions, resulting in vascular permeability. Notably, it was recently reported that ECs extracted from a primary tumor may exhibit some degree of stiffnessinsensitivity, shown by a stiffness-independent increase in basal Rho activity, traction force generation, and tubule formation. It is thought that this mechanism enables tumor-associated ECs to form networks, although leaky, in vivo

ECs can communicate through compliant substrates using a form of stiffness sensing in which cells sense directionally-biased increases in matrix stiffness due to the contractility of nearby cells [64]. From these results, a novel model emerged wherein cell-generated contraction forces induce a tension gradient in the surrounding ECM that activates the adhesion and migratory machinery of a second cell in a highly localized and polarized manner, resulting in durotaxis. Thus, durotaxis, first demonstrated by experimentally perturbing the substrate in the vicinity of a cell [58], is presumed to contribute to the cell spreading and migration that is characteristic of angiogenesis [47]. This result supports the finding that a balance between ECM stiffness and cellular traction forces determines the morphological features of endothelial networks [141].

Recently, it was shown that tumor-derived capillary endothelial cells exhibit some degree of stiffness-insensitivity similar to that which was described in epithelial cells [137]. These cells express high basal levels of Rho activity, thus preventing them from dynamically responding to the mechanical properties of their surrounding ECM. As expected, these cells exert higher traction forces, take on distinct cell morphologies, and assemble into tubular networks regardless of the mechanics of the underlying substrate or presence of other cells. However, normal mechanosensing endothelial cell behavior was restored upon ROCK inhibition. These results provide further evidence of the importance of both ECM stiffness and mechanosensitivity in maintaining normal tissue architecture and function. 


\subsubsection{Endothelium and Atherosclerosis: An Example of Cell Shape Correlating with Disease}

Forces in the Endothelium Determine Normal Tissue Structure and Function

In addition to matrix stiffness, there are a multitude of other forces that are present in the normal endothelial environment in vivo that influence cell adhesion and spreading, and thus, endothelial cell homeostasis and angiogenesis. Endothelial cells in vivo display a bi-axial hallmark of polarity (apical-basal and upstreamdownstream) in forming a monolayer that lines the interior of blood vessels. Here we will describe two significant mechanical cues inherent in the endothelial environment. The first is cyclic strain, which results from both the elasticity of blood vessels and the pulsatile nature of blood flow. The second is shear stress, which is exerted upon the endothelium as viscous blood flows over the adherent endothelial monolayer. Both of these mechanical cues influences the balance and localization of cell-cell and cell-matrix adhesions and alters cell shape to promote quiescence and thus aid in the maintenance of normal tissue function. In vivo, vessel circumference dynamically changes as a result of blood flow. This change in vessel circumference translates to cyclic strain along the axis perpendicular to the direction of fluid flow. This stretching induces a change in cell shape, accompanied by a re-distribution of matrix adhesions and stress fibers perpendicular to the axis of stretch. Critical to stress fiber maintenance and re-organization is the activity of the small GTPase Rho, without which cells are unable to properly orient stress fibers [80, 142]. Rho activity mediates actin dynamics required for cytoskeletal adaptation to maintain endothelial cell homeostasis. It is hypothesized that stress fibers form perpendicular to the direction of cyclic strain to reduce the net intracellular tension induced by stretch. The maintenance of stress fibers in this orientation through cytoskeletal adaptation has also been implicated in feedback to cellular signaling pathways that contribute to homeostasis [143]. Disruption of the physiological norm due to alterations in cyclic strain therefore has the potential to contribute to disease progression, particularly in increasing susceptibility to atherosclerosis development.

Fluid shear stress imposed by blood flow acts directly on the apical surface of endothelial cells, and similar to cyclic strain, contributes significantly to the geometrical anisotropy and cellular alignment observed in EC monolayers throughout the vasculature [143-145] (Fig. 7a). Because fluid shear stress (FSS)induced endothelial cell shape change is associated with cytoskeleton rearrangement [146, 147] and is tyrosine kinase-dependent [109], the Rho family of small GTPases and cell-generated forces have been heavily implicated in this cellular response [148]. Furthermore, the establishment and maintenance of the characteristic polarized morphology of ECs is adhesion-dependent and requires spatiotemporally-localized activation of intracellular signaling machinery [148, 149]. One of the earliest observable cellular responses to FSS acting on the apical surface is integrin activation on the basal surface due to an allosteric change that increases integrin affinity [150]. This activation and subsequent adhesion of 
Fig. 7 Physiological forces in the endothelium influence structure and function of the endothelium. a In healthy blood vessels, mechanical stimuli such as laminar blood flow and consistent radial cyclic strain determine cell polarity, satisfy EC homeostasis, and promote tissue quiescence. b Physiological factors such as disturbed blood flow and reduced radial strain disrupt EC mechanical homeostasis and are proatherogenic
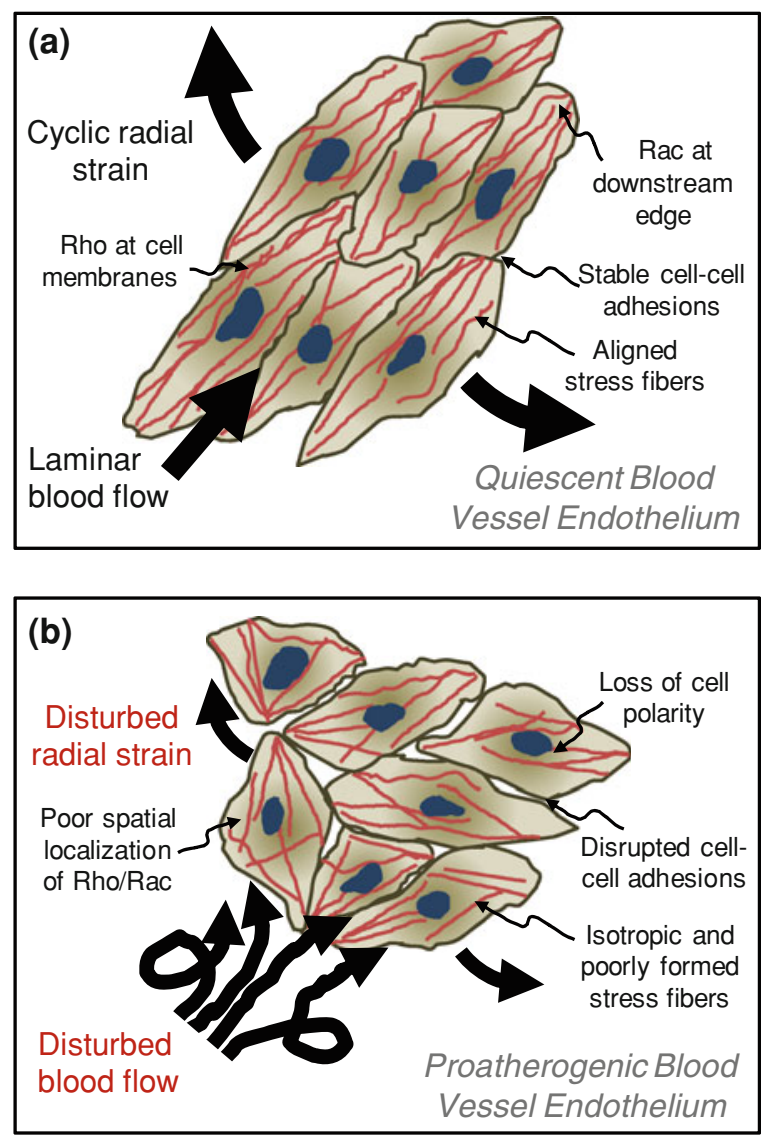

integrins results in rapid focal adhesion remodeling in the direction of the FSS [149], with increased FAK activation at the site of new focal adhesions [151]. Several groups have shown that regulation of intracellular signaling activity by shear stress occurs downstream of new integrin-ligand binding [150, 152-154], further emphasizing the importance of FSS-enhanced cell-matrix adhesion and focal adhesion turnover. Together, these localized biomolecular events contribute to the maintenance of the polarized cell shape shown in Fig. 7a.

In an elegant study by Wojciak-Stothard and Ridley [155], it was shown that cellular response to physiological shear stress occurs through regulation of Rhobased cellular contraction and Rac1-driven protrusion. The authors demonstrated that within 5 min of exposure to shear, endothelial cells exhibit rapid depolarization and contraction, produced by elevated RhoA-GTPase activity and actin stress fiber formation. Shortly thereafter, RhoA activity returns to basal levels as Rac1 activity increases, driving lamellipodial protrusion from the downstream side of cells in the direction of fluid flow. As cells achieve a spread and aligned morphology, Rac1-driven protrusion subsides while RhoA-based contractility and 
stress fiber formation increases, reestablishing tensional homeostasis with the underlying substrate. Several studies have confirmed that appropriate alignment and spreading in response to FSS depends upon dynamic regulation of RhoA activity [150, 155, 156] as well as local Rac1 activation within protruding lamellipodia [157, 158]. These FSS-induced effects are observed in the previously discussed middle and late stages of cell spreading, where Rac1 and RhoA have been implicated, respectively. Moreover, EC traction forces-which represent the biophysical manifestation of biomolecular events such as cell-matrix adhesions, intracellular signaling, and cytoskeletal dynamics-were increased and localized along the upstream and downstream edges of the cell in response to FSS, enabling cell shape change and realignment in flow [159].

One of the atheroprotective roles of FSS in vivo is to enable appropriate and efficient tissue maintenance and repair through control of cell shape and polarity. Investigating the time course of shear stress-regulated cell shape, Remuzzi et al. [146] showed that bovine aortic endothelial cells lose their polarized morphology $20 \mathrm{~h}$ after removal of flow. After $72 \mathrm{~h}$ in this static condition, FSS-conditioned cells reverted back to control (unstressed) levels of spreading, but still contained some aligned cytoskeletal components [146]. These results indicate that shear stress imposed by hemodynamic blood flow is essential for maintaining physiological endothelial cell morphology and illustrate how the loss or disruption of flow could have significant implications in pathology. Tissue vessel architecture relies not only upon moderation of cell-matrix adhesions, but also FSS-dependent maintenance of intercellular junctions, which preserve the barrier function of endothelial tissues [160, 161]. EC junctions, like cell-matrix adhesions, are intimately involved with the cytoskeleton, and are in fact regulated by localized Rac and Rho activity, which act antagonistically to stabilize and perturb EC junctions, respectively [155, 162].

Endothelial cell migration is not observed in quiescent blood vessels, but has been observed in disease states. As previously discussed, cell migration can be seen as cell shape change with a bias, and indeed, the forces that determine endothelial cell spreading and shape also contribute to EC migration. Cell migration during in vivo wound healing, in particular, is fluid shear stress-directed $[163,164]$. In vitro, both EC orientation and migration increase in response to physiological fluid shear stress in a scrape wound assay [165]. Likewise, Vyalov et al. [164] demonstrated that the healing response of wounded endothelial monolayers exposed to reduced shear stress in vivo is abnormal and insufficient. Thus, fluid shear stress likely plays a dual role in improving the efficiency of wound healing. First, FSS continually maintains ECs in a highly polarized state, thus equipping them to migrate immediately upon disruption of an adjacent cell-cell contact or other injury. This polarized morphology enhances the directional persistence of EC migration through Rac-induced alignment of the cytoskeleton and FSS-oriented cell spreading [166]. Second, as previously discussed, FSS causes increased Rho activation and thus, traction force generation, at the leading edge and rear of a cell, suggesting that FSS promotes a migratory phenotype [159]. Moreover, there is evidence that the biochemical composition of the basement 
membrane provides additional cues that influence stress fiber formation, cell spreading, and migration that follow injury in vitro [167].

\section{Mechanical Properties of the Basement Membrane and ECM}

It is becoming increasingly apparent that, in addition to fluid shear stress, the mechanical properties of endothelial ECM contribute to its adhesion and spreading, and thus, the establishment and maintenance of cellular homeostasis. Matrix mechanical properties affect all generations of the vascular system, from quiescent ECs lining large arteries to branching ECs during angiogenesis. The fibrillar nature of the ECM allows it to impart mechanical forces on cells through its intrinsic mechanical properties and transmitted forces, like those due to cyclic strain, in established blood vessels [143, 168, 169].

Perturbation of Normal Endothelial Mechanical Homeostasis and Cell Spreading

Atherosclerosis is a vascular disease characterized by the development of plaques or lesions within blood vessels involving proliferation of the vascular smooth muscle layer, thrombus formation, and accumulation of inflammatory mediators [170]. Atherosclerosis progression depends on multiple factors including genetic predisposition, dietary factors and lipid metabolism [170]. In the past two decades it has become apparent that the loss of endothelial mechanical homeostasis (due to factors including aberrant cyclic strain or fluid shear stress) also plays a significant role in the progression of cardiovascular disease. The cellular and molecular processes involved are highly complex and are strongly influenced by mechanical forces and cell shape.

Physiological FSS is atheroprotective, as it contributes to the maintenance of endothelial cell polarity, orientation, cell-cell adhesions, and thus, homeostasis $[143,171]$. Regions of the vasculature that are most prone to early atherogenesis are often exposed to disrupted flow. Disruptions primarily occur at points of vascular branching or bifurcations, where flow may become oscillatory (change direction) [172]. Additionally, the architecture of these bifurcations can result in altered magnitude and direction of the cyclic strain exerted on the endothelial layer, which can also be proatherogenic [143] (Fig. 7b). Interestingly, cell shape is one of the most obvious phenotypic differences between endothelial cells in atheroprone versus atheroprotective regions: endothelial cells in these atheroprone areas tend to be square in comparison to the elongated morphology seen in straight portions of the vessel. The ramifications of changes in cell shape in response to flow, including cytoskeletal and adhesion dynamics that permit minimization of flow resistance, have been under active investigation for a number of years [173-175].

Notably, there also is direct evidence that endothelial cell contractility and shape mediated through Rho signaling also contributes to endothelial permeability. 
This suggests a mechanism of cross-talk between fluid shear stress and EC contractility through the Rho pathway to affect cell structure and function.

\section{Summary}

Cells modulate their cytoskeleton to generate forces, which, when coordinated with cell-matrix and cell-cell adhesion regulation, enable cell shape change. This force is born in the molecular-level forces generated by actin polymerization and actin-myosin interactions that drive the membrane outward during spreading and stabilize cell shape of spread cells. Attention has been paid to the early process of spreading because it is a tractable, simplified model of the multistep integrated process of migration. Moreover, cell adhesion, the extent of spreading and cell shape have been shown to determine cell fate and function and drive several other adaptive cellular processes such as migration, growth and proliferation. Therefore knowledge of the biochemical and biophysical mechanism involved in inducing or controlling spreading will lead to greater insight into the factors controlling cellular homeostasis. While multiple examples exist where cell shape and spreading become disrupted during disease, as in cancer and atherosclerosis, there remains much to be learned about the inside-out and outside-in signals present during cell spreading and adhesion that help establish cellular homeostasis.

\section{References}

1. Pollard, T.D., Borisy, G.G.: Cellular motility driven by assembly and disassembly of actin filaments. Cell 112(4), 453-465 (2003)

2. Bindschadler, M., McGrath, J.L.: Formin' new ideas about actin filament generation. Proc. Natl Acad. Sci. USA 101(41):14685-14686 (2004)

3. Mitra, S.K., Hanson, D.A., Schlaepfer D.D.: Focal adhesion kinase: in command and control of cell motility. Nat. Rev. Mol. Cell. Biol. 6(1), 56-68 (2005)

4. Zhang, X., et al.: Talin depletion reveals independence of initial cell spreading from integrin activation and traction. Nat. Cell Biol. 10(9), 1062-1068 (2008)

5. Schwartz, I.M., et al.: The role of substrate curvature in actin-based pushing forces. Curr. Biol. 14(12):1094-1098 (2004)

6. Parekh, S.H., et al.: Loading history determines the velocity of actin-network growth. Nat. Cell Biol. 7(12):1219-1223 (2005)

7. Taubenberger, A., et al.: Revealing early steps of alpha2beta1 integrin-mediated adhesion to collagen type I by using single-cell force spectroscopy. Mol. Biol. Cell 18(5), 1634-1644 (2007)

8. Alon, R., et al.: Alpha4beta1-dependent adhesion strengthening under mechanical strain is regulated by paxillin association with the alpha4-cytoplasmic domain. J. Cell Biol. 171(6), 1073-1084 (2005)

9. Zhang, X., et al.: Molecular basis for the dynamic strength of the integrin alpha4beta1/ VCAM-1 interaction. Biophys. J. 87(5), 3470-3478 (2004)

10. Li, F., et al.: Force measurements of the alpha5betal integrin-fibronectin interaction. Biophys. J. 84(2 Pt 1), 1252-1262 (2003) 
11. Sun, Z., et al.: Mechanical properties of the interaction between fibronectin and alpha5beta1-integrin on vascular smooth muscle cells studied using atomic force microscopy. Am. J. Physiol. Heart Circ. Physiol. 289(6), H2526-H2535 (2005)

12. Trache, A., Meininger, G.A.: Atomic force-multi-optical imaging integrated microscope for monitoring molecular dynamics in live cells. J. Biomed. Opt. 10(6), 064023 (2005)

13. Thie, M., et al.: Interactions between trophoblast and uterine epithelium: monitoring of adhesive forces. Hum. Reprod. 13(11):3211-3219 (1998)

14. Zhang, X., Wojcikiewicz, E., Moy, V.T.: Force spectroscopy of the leukocyte functionassociated antigen-1/intercellular adhesion molecule-1 interaction. Biophys. J. 83(4), 22702279 (2002)

15. Zhang, X., Wojcikiewicz, E.P., Moy V.T.: Dynamic adhesion of $\mathrm{T}$ lymphocytes to endothelial cells revealed by atomic force microscopy. Exp. Biol. Med. (Maywood) 231(8), 1306-1312 (2006)

16. Wojcikiewicz, E.P., et al.: Force spectroscopy of LFA-1 and its ligands, ICAM-1 and ICAM-2. Biomacromolecules 7(11), 3188-3195 (2006)

17. Lehenkari, P.P., Horton, M.A.: Single integrin molecule adhesion forces in intact cells measured by atomic force microscopy. Biochem. Biophys. Res. Commun. 259(3), 645-650 (1999)

18. Prass, M., et al.: Direct measurement of the lamellipodial protrusive force in a migrating cell. J. Cell Biol. 174(6), 767-772 (2006)

19. Gittes, F., et al.: Flexural rigidity of microtubules and actin filaments measured from thermal fluctuations in shape. J. Cell Biol. 120(4), 923-934 (1993)

20. Isambert, H., et al.: Flexibility of actin filaments derived from thermal fluctuations. Effect of bound nucleotide, phalloidin, and muscle regulatory proteins. J. Biol. Chem. 270(19), 11437-11444 (1995)

21. McCullough, B.R., et al.: Cofilin increases the bending flexibility of actin filaments: implications for severing and cell mechanics. J. Mol. Biol. 381(3), 550-558 (2008)

22. Pfaendtner, J., et al.: Structure and dynamics of the actin filament. J. Mol. Biol. 396(2), 252-263

23. Cai, Y., et al.: Cytoskeletal coherence requires myosin-IIA contractility. J. Cell Sci. 123 (Pt 3), 413-423

24. Finer, J.T., Simmons, R.M., Spudich, J.A.: Single myosin molecule mechanics: piconewton forces and nanometre steps. Nature 368(6467), 113-119 (1994)

25. Dogterom, M., Yurke, B.: Measurement of the force-velocity relation for growing microtubules. Science 278(5339), 856-860 (1997)

26. Kerssemakers, J.W., et al.: Assembly dynamics of microtubules at molecular resolution. Nature 442(7103), 709-712 (2006)

27. Wang, N., et al.: Mechanical behavior in living cells consistent with the tensegrity model. Proc. Natl Acad. Sci. USA 98(14), 7765-7770 (2001)

28. Brangwynne, C.P., et al.: Microtubules can bear enhanced compressive loads in living cells because of lateral reinforcement. J. Cell Biol. 173(5), 733-741 (2006)

29. Herrmann, H., et al.: Intermediate filaments: from cell architecture to nanomechanics. Nat. Rev. Mol. Cell. Biol. 8(7), 562-573 (2007)

30. Wagner, O.I., et al.: Softness, strength and self-repair in intermediate filament networks. Exp. Cell Res. 313(10), 2228-2235 (2007)

31. Macpherson, I., Montagnier, L.: Agar Suspension Culture for the Selective Assay of Cells Transformed by Polyoma Virus. Virology 23, 291-294 (1964)

32. Stoker, M., et al.: Anchorage and growth regulation in normal and virus-transformed cells. Int. J. Cancer 3(5), 683-693 (1968)

33. Yamada, K.M., Yamada, S.S., Pastan, I.: Cell surface protein partially restores morphology, adhesiveness, and contact inhibition of movement to transformed fibroblasts. Proc. Natl Acad. Sci. USA 73(4), 1217-1221 (1976)

34. Ali, I.U., et al.: Restoration of normal morphology, adhesion and cytoskeleton in transformed cells by addition of a transformation-sensitive surface protein. Cell 11(1), 115-126 (1977) 
35. Heggeness, M.H., Ash, J.F., Singer, S.J.: Transmembrane linkage of fibronectin to intracellular actin-containing filaments in cultured human fibroblasts. Ann. N. Y. Acad. Sci. 312: 414-417 (1978)

36. Ali, I.U., Hynes, R.O.: Effects of LETS glycoprotein on cell motility. Cell 14(2), 439-446 (1978)

37. Hynes, R.O., Destree, A.T.: Relationships between fibronectin (LETS protein) and actin. Cell 15(3), 875-886 (1978)

38. Folkman, J., Moscona, A.: Role of cell shape in growth control. Nature 273(5661), 345-349 (1978)

39. Tamkun, J.W., et al.: Structure of integrin, a glycoprotein involved in the transmembrane linkage between fibronectin and actin. Cell 46(2), 271-282 (1986)

40. Argraves, W.S., et al.: cDNA sequences from the alpha subunit of the fibronectin receptor predict a transmembrane domain and a short cytoplasmic peptide. J. Biol. Chem. 261(28), 12922-12924 (1986)

41. Miranti, C.K., Brugge, J.S.: Sensing the environment: a historical perspective on integrin signal transduction. Nat. Cell Biol. 4(4), E83-E90 (2002)

42. Arnaout, M.A., Mahalingam, B., Xiong, J.P.: Integrin structure, allostery, and bidirectional signaling. Annu. Rev. Cell Dev. Biol. 21:381-410 (2005)

43. van Kooyk, Y., Figdor, C.G.: Avidity regulation of integrins: the driving force in leukocyte adhesion. Curr. Opin. Cell Biol. 12(5), 542-547 (2000)

44. Galbraith, C.G., Yamada, K.M., Sheetz, M.P.: The relationship between force and focal complex development. J. Cell Biol. 159(4), 695-705 (2002)

45. Helenius, J., et al.: Single-cell force spectroscopy. J. Cell Sci. 121(Pt 11), 1785-1791 (2008)

46. Galbraith, C.G., Sheetz, M.P.: Forces on adhesive contacts affect cell function. Curr. Opin. Cell Biol. 10(5), 566-571 (1998)

47. Califano, J.P., Reinhart-King, C.A.: A Balance of Substrate Mechanics and Matrix Chemistry Regulates Endothelial Cell Network Assembly. Cell. Mol. Bioeng. 1, 122-132 (2008)

48. Balaban, N.Q., et al.: Force and focal adhesion assembly: a close relationship studied using elastic micropatterned substrates. Nat. Cell Biol. 3(5), 466-472 (2001)

49. Zamir, E., Geiger, B.: Molecular complexity and dynamics of cell-matrix adhesions. J. Cell Sci. 114(Pt 20), 3583-3590 (2001)

50. Riveline, D., et al.: Focal contacts as mechanosensors: externally applied local mechanical force induces growth of focal contacts by an mDia1-dependent and ROCK-independent mechanism. J. Cell Biol. 153(6), 1175-1186 (2001)

51. Puklin-Faucher, E., Sheetz, M.P.: The mechanical integrin cycle. J. Cell Sci. 122(Pt 2), 179 186 (2009)

52. Discher, D.E., Janmey, P., Wang, Y.L.: Tissue cells feel and respond to the stiffness of their substrate. Science 310(5751), 1139-1143 (2005)

53. Shyy, J.Y., Chien, S.: Role of integrins in cellular responses to mechanical stress and adhesion. Curr. Opin. Cell Biol. 9(5), 707-713 (1997)

54. Hamill, O.P., Martinac, B.: Molecular basis of mechanotransduction in living cells. Physiol. Rev. 81(2), 685-740 (2001)

55. Ingber, D.E.: Cellular mechanotransduction: putting all the pieces together again. FASEB J. 20(7), 811-827 (2006)

56. Pelham, R.J., Jr, Wang, Y.: Cell locomotion and focal adhesions are regulated by substrate flexibility. Proc. Natl Acad. Sci. USA 94(25), 13661-13665 (1997)

57. Choquet, D., Felsenfeld, D.P., Sheetz, M.P.: Extracellular matrix rigidity causes strengthening of integrin-cytoskeleton linkages. Cell 88(1), 39-48 (1997)

58. Lo, C.M., et al.: Cell movement is guided by the rigidity of the substrate. Biophys. J. 79(1), 144-152 (2000)

59. Engler, A.J., et al.: Matrix elasticity directs stem cell lineage specification. Cell 126(4), 677-689 (2006) 
60. Tomasek, J.J., et al.: Myofibroblasts and mechano-regulation of connective tissue remodelling. Nat. Rev. Mol. Cell. Biol. 3(5), 349-363 (2002)

61. Engler, A.J., et al.: Myotubes differentiate optimally on substrates with tissue-like stiffness: pathological implications for soft or stiff microenvironments. J. Cell Biol. 166(6), 877-887 (2004)

62. Yeung, T., et al.: Effects of substrate stiffness on cell morphology, cytoskeletal structure, and adhesion. Cell Motil. Cytoskeleton 60(1), 24-34 (2005)

63. Guo, W.H., et al.: Substrate rigidity regulates the formation and maintenance of tissues. Biophys. J. 90(6), 2213-2220 (2006)

64. Reinhart-King, C.A., Dembo, M., Hammer, D.A.: Cell-cell mechanical communication through compliant substrates. Biophys. J. 95(12), 6044-6051 (2008)

65. Califano, J.P., Reinhart-King, C.A.: The effects of substrate elasticity on endothelial cell network formation and traction force generation. Conf. Proc. IEEE Eng. Med. Biol. Soc. 2009, 3343-3345 (2009)

66. Paszek, M.J., et al.: Tensional homeostasis and the malignant phenotype. Cancer Cell 8(3), 241-254 (2005)

67. Provenzano, P.P., et al.: Matrix density-induced mechanoregulation of breast cell phenotype, signaling and gene expression through a FAK-ERK linkage. Oncogene 28(49), 4326-4343 (2009)

68. Brown, R.A., et al.: Tensional homeostasis in dermal fibroblasts: mechanical responses to mechanical loading in three-dimensional substrates. J. Cell Physiol. 175(3), 323-332 (1998)

69. Chen, C.S.: Mechanotransduction - a field pulling together? J. Cell Sci. 121(Pt 20), 3285$3292(2008)$

70. Giannone, G., et al.: Periodic lamellipodial contractions correlate with rearward actin waves. Cell 116(3), 431-443 (2004)

71. Dubin-Thaler, B.J., et al.: Nanometer analysis of cell spreading on matrix-coated surfaces reveals two distinct cell states and STEPs. Biophys. J. 86(3), 1794-1806 (2004)

72. Dobereiner, H.G., et al.: Dynamic phase transitions in cell spreading. Phys. Rev. Lett. 93(10), 108105 (2004)

73. Dobereiner, H.G., et al.: Force sensing and generation in cell phases: analyses of complex functions. J. Appl. Physiol. 98(4), 1542-1546 (2005)

74. Cuvelier, D., et al.: The universal dynamics of cell spreading. Curr. Biol. 17(8), 694-699 (2007)

75. McGrath, J.L.: Cell spreading: the power to simplify. Curr. Biol. 17(10), R357-R358 (2007)

76. Dubin-Thaler, B.J., et al.: Quantification of cell edge velocities and traction forces reveals distinct motility modules during cell spreading. PLoS One 3(11), e3735 (2008)

77. Cai, Y., et al.: Nonmuscle myosin IIA-dependent force inhibits cell spreading and drives F-actin flow. Biophys. J. 91(10), 3907-3920 (2006)

78. Bell, G.I., Dembo, M., Bongrand, P.: Cell adhesion. Competition between nonspecific repulsion and specific bonding. Biophys. J. 45(6), 1051-1064 (1984)

79. Torney, D.C., Dembo, M., Bell, G.I.: Thermodynamics of cell adhesion. II. Freely mobile repellers. Biophys. J. 49(2), 501-507 (1986)

80. Huveneers, S., Danen, E.H.: Adhesion signaling - crosstalk between integrins, Src and Rho. J. Cell Sci. 122(Pt 8), 1059-1069 (2009)

81. Reinhart-King, C.A., Dembo, M., Hammer, D.A.: The dynamics and mechanics of endothelial cell spreading. Biophys. J. 89(1), 676-689 (2005)

82. Bhadriraju, K., et al.: Activation of ROCK by RhoA is regulated by cell adhesion, shape, and cytoskeletal tension. Exp. Cell Res. 313(16), 3616-3623 (2007)

83. Stamenovic, D., et al.: Cell prestress. II. Contribution of microtubules. Am. J. Physiol. Cell Physiol. 282(3), C617-C624 (2002)

84. Mejillano, M.R., et al.: Lamellipodial versus filopodial mode of the actin nanomachinery: pivotal role of the filament barbed end. Cell 118(3), 363-373 (2004)

85. Sheetz, M.P., Wayne, D.B., Pearlman, A.L.: Extension of filopodia by motor-dependent actin assembly. Cell Motil. Cytoskeleton 22(3), 160-169 (1992) 
86. Giannone, G., et al.: Lamellipodial actin mechanically links myosin activity with adhesionsite formation. Cell 128(3), 561-575 (2007)

87. Wang, N., et al.: Micropatterning tractional forces in living cells. Cell Motil. Cytoskeleton 52(2), 97-106 (2002)

88. Ren, X.D., et al.: Focal adhesion kinase suppresses Rho activity to promote focal adhesion turnover. J. Cell Sci. 113(Pt 20), 3673-3678 (2000)

89. White, D.P., Caswell, P.T., Norman, J.C.: alpha v beta3 and alpha5beta1 integrin recycling pathways dictate downstream Rho kinase signaling to regulate persistent cell migration. J. Cell Biol. 177(3), 515-525 (2007)

90. Schoenwaelder, S.M., Burridge, K.: Bidirectional signaling between the cytoskeleton and integrins. Curr. Opin. Cell Biol. 11(2), 274-286 (1999)

91. Hotulainen, P., Lappalainen, P.: Stress fibers are generated by two distinct actin assembly mechanisms in motile cells. J. Cell Biol. 173(3), 383-394 (2006)

92. Reinhart-King, C.A., Dembo, M., Hammer, D.A.: Endothelial Cell Traction Forces on RGD-Derivatized Polyacrylamide Substrata. Langmuir 19(5), 1573-1579 (2003)

93. Plopper, G., Ingber, D.E.: Rapid induction and isolation of focal adhesion complexes. Biochem. Biophys. Res. Commun. 193(2), 571-578 (1993)

94. Schmidt, C.E., et al.: Integrin-cytoskeletal interactions in migrating fibroblasts are dynamic, asymmetric, and regulated. J. Cell Biol. 123(4), 977-991 (1993)

95. Gumbiner, B.M.: Cell adhesion: the molecular basis of tissue architecture and morphogenesis. Cell 84(3), 345-357 (1996)

96. Spiegelman, B.M., Ginty, C.A.: Fibronectin modulation of cell shape and lipogenic gene expression in 3T3-adipocytes. Cell 35(3 Pt 2), 657-666 (1983)

97. Chen, C.S., et al.: Geometric control of cell life and death. Science 276(5317), 1425-1428 (1997)

98. McBeath, R., et al.: Cell shape, cytoskeletal tension, and RhoA regulate stem cell lineage commitment. Dev. Cell 6(4), 483-495 (2004)

99. Vogel, V., Sheetz, M.: Local force and geometry sensing regulate cell functions. Nat. Rev. Mol. Cell. Biol. 7(4), 265-275 (2006)

100. Lauffenburger, D.A., Horwitz, A.F.: Cell migration: a physically integrated molecular process. Cell 84(3), 359-369 (1996)

101. Ridley, A.J., et al.: Cell migration: integrating signals from front to back. Science 302(5651), 1704-1709 (2003)

102. Guo, W.H., Wang, Y.L.: Retrograde fluxes of focal adhesion proteins in response to cell migration and mechanical signals. Mol. Biol. Cell 18(11), 4519-4527 (2007)

103. Friedl, P., Wolf, K.: Plasticity of cell migration: a multiscale tuning model. J. Cell Biol. 188(1), 11-19 (2010)

104. Sheetz, M.P., Felsenfeld, D.P., Galbraith, C.G.: Cell migration: regulation of force on extracellular-matrix-integrin complexes. Trends Cell Biol. 8(2), 51-54 (1998)

105. Paszek, M.J., Weaver, V.M.: The tension mounts: mechanics meets morphogenesis and malignancy. J Mammary Gland Biol. Neoplasia 9(4), 325-342 (2004)

106. Bentley, S.A.: Bone marrow connective tissue and the haemopoietic microenvironment. Br. J. Haematol. 50(1), 1-6 (1982)

107. Grigoriadis, A.E., Heersche, J.N., Aubin, J.E.: Differentiation of muscle, fat, cartilage, and bone from progenitor cells present in a bone-derived clonal cell population: effect of dexamethasone. J. Cell Biol. 106(6), 2139-2151 (1988)

108. Howlett, A.R., Bissell, M.J.: The influence of tissue microenvironment (stroma and extracellular matrix) on the development and function of mammary epithelium. Epithelial Cell Biol. 2(2), 79-89 (1993)

109. Malek, A.M., Izumo, S.: Mechanism of endothelial cell shape change and cytoskeletal remodeling in response to fluid shear stress. J. Cell Sci. 109 (Pt 4), 713-726 (1996)

110. Gregoire, F.M., Smas, C.M., Sul, H.S.: Understanding adipocyte differentiation. Physiol. Rev. 78(3), 783-809 (1998) 
111. Polte, T.R., et al.: Extracellular matrix controls myosin light chain phosphorylation and cell contractility through modulation of cell shape and cytoskeletal prestress. Am. J. Physiol. Cell Physiol. 286(3), C518-C528 (2004)

112. Bissell, M.J., Rizki, A., Mian, I.S.: Tissue architecture: the ultimate regulator of breast epithelial function. Curr. Opin. Cell Biol. 15(6), 753-762 (2003)

113. Drubin, D.G., Nelson, W.J.: Origins of cell polarity. Cell 84(3), 335-344 (1996)

114. Gumbiner, B.M.: Regulation of cadherin-mediated adhesion in morphogenesis. Nat. Rev. Mol. Cell. Biol. 6(8), 622-634 (2005)

115. Kass, L., et al.: Mammary epithelial cell: influence of extracellular matrix composition and organization during development and tumorigenesis. Int. J. Biochem. Cell Biol. 39(11), 1987-1994 (2007)

116. Wozniak, M.A., et al.: ROCK-generated contractility regulates breast epithelial cell differentiation in response to the physical properties of a three-dimensional collagen matrix. J. Cell Biol. 163(3), 583-595 (2003)

117. Moore, K.A., et al.: Control of basement membrane remodeling and epithelial branching morphogenesis in embryonic lung by Rho and cytoskeletal tension. Dev. Dyn. 232(2), 268$281(2005)$

118. Wang, A.Z., Ojakian, G.K., Nelson, W.J.: Steps in the morphogenesis of a polarized epithelium. I. Uncoupling the roles of cell-cell and cell-substratum contact in establishing plasma membrane polarity in multicellular epithelial (MDCK) cysts. J. Cell Sci. 95( $\mathrm{Pt} 1)$, 137-151 (1990)

119. Szauter, K.M., et al.: Lysyl oxidase in development, aging and pathologies of the skin. Pathol. Biol. (Paris) 53(7), 448-456 (2005)

120. Padera, T.P., et al.: Pathology: cancer cells compress intratumour vessels. Nature 427(6976), 695 (2004)

121. Baker, E.L., Bonnecaze, R.T., Zaman, M.H.: Extracellular matrix stiffness and architecture govern intracellular rheology in cancer. Biophys. J. 97(4), 1013-1021 (2009)

122. Bellizzi, A., et al.: RhoA protein expression in primary breast cancers and matched lymphocytes is associated with progression of the disease. Int. J. Mol. Med. 22(1), 25-31 (2008)

123. Burbelo, P., Wellstein, A., Pestell, R.G.: Altered Rho GTPase signaling pathways in breast cancer cells. Breast Cancer Res. Treat. 84(1), 43-48 (2004)

124. Khaled, W., et al.: Palpation imaging using a haptic system for virtual reality applications in medicine. Stud. Health Technol. Inform. 98, 147-153 (2004)

125. Park, C.C., et al.: Beta1 integrin inhibitory antibody induces apoptosis of breast cancer cells, inhibits growth, and distinguishes malignant from normal phenotype in three dimensional cultures and in vivo. Cancer Res. 66(3), 1526-1535 (2006)

126. Kumar, S., Weaver, V.M.: Mechanics, malignancy, and metastasis: the force journey of a tumor cell. Cancer Metastasis Rev. 28(1-2), 113-127 (2009)

127. Saez, A., et al.: Is the mechanical activity of epithelial cells controlled by deformations or forces? Biophys. J. 89(6), L52-L54 (2005)

128. Califano, J.P., Reinhart-King, C.A.: Exogenous and endogenous force regulation of endothelial cell behavior. J. Biomech. 43(1), 79-86

129. Fritz, G., Just, I., Kaina, B.: Rho GTPases are over-expressed in human tumors. Int. J. Cancer 81(5), 682-687 (1999)

130. Kleer, C.G., et al.: WISP3 and RhoC guanosine triphosphatase cooperate in the development of inflammatory breast cancer. Breast Cancer Res. 6(2), R110-R115 (2004)

131. Takaishi, K., et al.: Regulation of cell-cell adhesion by rac and rho small G proteins in MDCK cells. J. Cell Biol. 139(4), 1047-1059 (1997)

132. Braga, V.M., et al.: The small GTPases Rho and Rac are required for the establishment of cadherin-dependent cell-cell contacts. J. Cell Biol. 137(6), 1421-1431 (1997)

133. Levental, K.R., et al.: Matrix crosslinking forces tumor progression by enhancing integrin signaling. Cell 139(5), 891-906 (2009) 
134. Lee, J.M., et al.: The epithelial-mesenchymal transition: new insights in signaling, development, and disease. J. Cell Biol. 172(7), 973-981 (2006)

135. Zaman, M.H., et al.: Migration of tumor cells in 3D matrices is governed by matrix stiffness along with cell-matrix adhesion and proteolysis. Proc. Natl Acad. Sci. USA 103(29), 10889-10894 (2006)

136. Bos, J.L.: ras oncogenes in human cancer: a review. Cancer Res 49(17), 4682-4689 (1989)

137. Ghosh, K., et al.: Tumor-derived endothelial cells exhibit aberrant Rho-mediated mechanosensing and abnormal angiogenesis in vitro. Proc. Natl Acad. Sci. USA 105(32), 11305-11310 (2008)

138. Hida, K., Hida, Y., Shindoh, M.: Understanding tumor endothelial cell abnormalities to develop ideal anti-angiogenic therapies. Cancer Sci. 99(3), 459-466 (2008)

139. Carmeliet, P.: Angiogenesis in health and disease. Nat. Med. 9(6), 653-660 (2003)

140. Krishnan, L., et al.: Interaction of angiogenic microvessels with the extracellular matrix. Am. J. Physiol. Heart Circ. Physiol. 293(6), H3650-H3658 (2007)

141. Sieminski, A.L., Hebbel, R.P., Gooch, K.J.: The relative magnitudes of endothelial force generation and matrix stiffness modulate capillary morphogenesis in vitro. Exp. Cell Res. 297(2), 574-584 (2004)

142. Etienne-Manneville, S., Hall, A.: Rho GTPases in cell biology. Nature 420(6916), 629-635 (2002)

143. Chien, S.: Mechanotransduction and endothelial cell homeostasis: the wisdom of the cell. Am. J. Physiol. Heart Circ. Physiol. 292(3), H1209-H1224 (2007)

144. Levesque, M.J., Nerem, R.M.: The elongation and orientation of cultured endothelial cells in response to shear stress. J. Biomech. Eng. 107(4), 341-347 (1985)

145. Ives, C.L., Eskin, S.G., McIntire, L.V.: Mechanical effects on endothelial cell morphology: in vitro assessment. In Vitro Cell Dev. Biol. 22(9), 500-507 (1986)

146. Remuzzi, A., et al.: Orientation of endothelial cells in shear fields in vitro. Biorheology 21(4), 617-630 (1984)

147. Galbraith, C.G., Skalak, R., Chien, S.: Shear stress induces spatial reorganization of the endothelial cell cytoskeleton. Cell Motil. Cytoskeleton 40(4), 317-330 (1998)

148. Tzima, E.: Role of small GTPases in endothelial cytoskeletal dynamics and the shear stress response. Circ. Res. 98(2), 176-185 (2006)

149. Davies, P.F., Robotewskyj, A., Griem, M.L.: Quantitative studies of endothelial cell adhesion. Directional remodeling of focal adhesion sites in response to flow forces. J. Clin. Invest. 93(5), 2031-2038 (1994)

150. Tzima, E., et al.: Activation of integrins in endothelial cells by fluid shear stress mediates Rho-dependent cytoskeletal alignment. EMBO J. 20(17), 4639-4647 (2001)

151. Li, S., et al.: Fluid shear stress activation of focal adhesion kinase. Linking to mitogenactivated protein kinases. J. Biol. Chem. 272(48), 30455-30462 (1997)

152. Ishida, T., et al.: MAP kinase activation by flow in endothelial cells. Role of beta 1 integrins and tyrosine kinases. Circ. Res. 79(2), 310-316 (1996)

153. Jalali, S., et al.: Integrin-mediated mechanotransduction requires its dynamic interaction with specific extracellular matrix (ECM) ligands. Proc. Natl Acad. Sci. USA 98(3), 10421046 (2001)

154. Reyes-Reyes, M., et al.: Phosphatidylinositol 3-kinase mediates integrin-dependent NFkappaB and MAPK activation through separate signaling pathways. J. Cell Sci. 114( $\mathrm{Pt} 8)$, 1579-1589 (2001)

155. Wojciak-Stothard, B., Ridley, A.J.: Shear stress-induced endothelial cell polarization is mediated by Rho and Rac but not Cdc42 or PI 3-kinases. J. Cell Biol. 161(2), 429-439 (2003)

156. Li, S., et al.: Distinct roles for the small GTPases Cdc 42 and Rho in endothelial responses to shear stress. J. Clin. Invest. 103(8), 1141-1150 (1999)

157. Kraynov, V.S., et al.: Localized Rac activation dynamics visualized in living cells. Science 290(5490), 333-337 (2000) 
158. Tzima, E., et al.: Activation of Rac1 by shear stress in endothelial cells mediates both cytoskeletal reorganization and effects on gene expression. EMBO J. 21(24), 6791-6800 (2002)

159. Shiu, Y.T., et al.: Rho mediates the shear-enhancement of endothelial cell migration and traction force generation. Biophys. J. 86(4), 2558-2565 (2004)

160. Seebach, J., et al.: Endothelial barrier function under laminar fluid shear stress. Lab. Invest. 80(12), 1819-1831 (2000)

161. Shikata, Y., et al.: Differential effects of shear stress and cyclic stretch on focal adhesion remodeling, site-specific FAK phosphorylation, and small GTPases in human lung endothelial cells. Exp. Cell Res. 304(1), 40-49 (2005)

162. Wojciak-Stothard, B., et al.: Rho and Rac but not Cdc42 regulate endothelial cell permeability. J. Cell Sci. 114(Pt 7), 1343-1355 (2001)

163. Madri, J.A., Stenn, K.S.: Aortic endothelial cell migration. I. Matrix requirements and composition. Am. J. Pathol. 106(2), 180-186 (1982)

164. Vyalov, S., Langille, B.L., Gotlieb, A.I.: Decreased blood flow rate disrupts endothelial repair in vivo. Am. J. Pathol. 149(6), 2107-2118 (1996)

165. van der Meer, A.D., et al.: A microfluidic wound-healing assay for quantifying endothelial cell migration. Am. J. Physiol. Heart Circ. Physiol. 298(2), H719-H725 (2010)

166. Chung, C.Y., et al.: Role of Rac in controlling the actin cytoskeleton and chemotaxis in motile cells. Proc. Natl Acad. Sci. USA 97(10), 5225-5230 (2000)

167. Young, W.C., Herman, I.M.: Extracellular matrix modulation of endothelial cell shape and motility following injury in vitro. J. Cell Sci. 73, 19-32 (1985)

168. Iba, T., Sumpio, B.E.: Morphological response of human endothelial cells subjected to cyclic strain in vitro. Microvasc. Res. 42(3), 245-254 (1991)

169. Kalluri, R.: Basement membranes: structure, assembly and role in tumour angiogenesis. Nat. Rev. Cancer 3(6), 422-433 (2003)

170. Singh, R.B., et al.: Pathogenesis of atherosclerosis: a multifactorial process. Exp. Clin. Cardiol. 7(1), 40-53 (2002)

171. Choi, C.K., Helmke, B.P.: Short-term shear stress induces rapid actin dynamics in living endothelial cells. Mol. Cell. Biomech. 5(4), 247-258 (2008)

172. Garin, G., Berk, B.C.: Flow-mediated signaling modulates endothelial cell phenotype. Endothelium 13(6), 375-384 (2006)

173. Chiu, Y.J., et al.: Endothelial cell-cell adhesion and mechanosignal transduction. Endothelium 11(1), 59-73 (2004)

174. Goldfinger, L.E., et al.: Localized alpha4 integrin phosphorylation directs shear stressinduced endothelial cell alignment. Circ. Res. 103(2), 177-185 (2008)

175. Helmke, B.P.: Choosing sides in polarized endothelial adaptation to shear stress. Circ. Res. 103(2), 122-124 (2008) 